\title{
Mom technology and volumes of hyperbolic 3-manifolds
}

\author{
David Gabai, ${ }^{1}$ Robert Meyerhoff ${ }^{2}$, and Peter Milley ${ }^{3}$
}

\begin{abstract}
This paper introduces Mom technology to understand low volume hyperbolic 3manifolds; it is used in [GMM3] and [M1] to show that the Weeks manifold is the unique closed orientable hyperbolic 3-manifold of least volume. Here we enumerate the hyperbolic Mom- $n$ manifolds for $n \leq 3$, offer a conjectural enumeration when $n=4$, and establish important technical results about embedding hyperbolic Mom- $n$ manifolds into hyperbolic 3-manifolds.
\end{abstract}

Mathematics Subject Classification (2010). Primary 57M50; Secondary 51M10, 51M25.

Keywords. Mom technology, hyperbolic 3-manifolds, volume.

\section{Introduction}

This paper introduces a new technique, Mom technology, to understand the structure of low-volume complete orientable hyperbolic 3-manifolds. Among other things, we enumerate the 21 Mom- $n, n \leq 3$ hyperbolic 3-manifolds and describe the 138 conjectural Mom-4 manifolds.

This work is used in [GMM3] to give a positive solution to the long-standing conjecture that the Weeks manifold is the unique lowest volume closed orientable hyperbolic 3-manifold. It is used in [LM] to solve affirmatively two conjectures of Cameron Gordon. The first states that, given a complete finite-volume hyperbolic 3-manifold with boundary a torus, the maximal number of exceptional slopes (these correspond to non-hyperbolic Dehn surgeries) is 10. The second states that, further, the maximal intersection number of exceptional slopes is 8 .

William Thurston has long promoted the idea that volume is a good measure of the complexity of a hyperbolic 3-manifold (see, for example, [Th1] page 6.48). Among known low-volume manifolds, Jeff Weeks ([We]) and independently Sergei Matveev and Anatoly Fomenko ([MF]) have observed that there is a close connection between the volume of closed hyperbolic 3-manifolds and combinatorial complexity. One

\footnotetext{
${ }^{1}$ Partially supported by NSF grants DMS-0346270, DMS-0504110 and DMS-1006553.

${ }^{2}$ Partially supported by NSF grant 0553787.

${ }^{3}$ Partially supported by NSF grant DMS-0554624 and ARC Discovery grant DP0663399.
} 
goal of this paper is to begin to explain, in an organized fashion, this phenomenon as summarized by the following:

Hyperbolic Complexity Conjecture 0.1 (Thurston, Weeks, Matveev-Fomenko). The complete low-volume hyperbolic 3-manifolds can be obtained by filling cusped hyperbolic 3-manifolds of small topological complexity.

Remark 0.2. Part of the challenge of this conjecture is to clarify the undefined adjectives low and small. We propose that the manifolds obtained by Dehn filling a Mom- $n, n \leq 3$ (or $n \leq 4$ ) include all the reasonably low volume complete hyperbolic 3-manifolds. Indeed, in [GMM3] we show that the 10 lowest volume 1-cusped hyperbolic 3-manifolds (or equivalently the manifolds of volume $\leq 2.848$ ) are obtained by filling a Mom- $n$ manifold with $n \leq 3$. Furthermore, based on computer calculation all the cusped orientable manifolds in the SnapPea census of volume $\leq 3.177$, $\leq 4.059$, and $\leq 5.468$ are obtained by respectively filling Mom-2, Mom-3, and Mom-4 manifolds. (This includes 2948 manifolds.)

In the late 1970s, Troels Jorgensen proved that for any positive constant $C$ there is a finite collection of cusped hyperbolic 3-manifolds from which all complete hyperbolic 3-manifolds of volume less than or equal to $C$ can be obtained by Dehn filling. If it could be proved that all cusped manifolds of volume $\leq 5.0$ can be obtained by filling a Mom- $n$ manifold with $n \leq 4$, and if a corresponding result (with a somewhat lower volume bound) could be proved for closed manifolds, then we would have a concrete and satisfying realization of Jorgensen's Theorem for "low" values of $C$.

A special case of the Hyperbolic Complexity Conjecture is the following:

Theorem 0.3 ([GMM3]). The Weeks manifold $M_{W}$, obtained by $(5,1),(5,2)$ surgery on the two components of the Whitehead link, is the unique orientable hyperbolic 3manifold of minimum volume.

Note that the volume of $M_{W}$ is $0.942 \ldots$ The proof required understanding all the very low volume manifolds obtained by filling a Mom- $n$ manifold for $n \leq 3$, an analysis carried out in [M1].

All manifolds in this paper will be orientable and all hyperbolic structures are complete. We call a compact manifold hyperbolic if its interior supports a complete hyperbolic structure of finite volume. Unless said otherwise, all compact hyperbolic 3-manifolds in this paper are compactified cusped hyperbolic 3-manifolds.

Definition 0.4. A Mom- $n$ structure $(M, T, \Delta)$ consists of a compact 3-manifold $M$ whose boundary is a union of tori, a preferred boundary component $T$, and a handle decomposition $\Delta$ of the following type. Starting from $T \times I, n$ 1-handles and $n$ 
2-handles are attached to $T \times 1$ such that each 2-handle goes over exactly three 1handles, counted with multiplicity. Furthermore, each 1-handle encounters at least two 2-handles, counted with multiplicity. We say that $M$ is a Mom- $n$ if it possesses a Mom- $n$ structure $(M, T, \Delta)$.

Remark 0.5. On a Mom- $n$, the handle decomposition $\Delta$ deformation retracts to an almost simple 2-complex which has $2 n$ true vertices, in the sense of Matveev [Mv2]. Therefore Mom- $n$ manifolds are a subset of those with Matveev complexity at most $2 n$.

Here is the fundamental idea at the foundation of our project. Given a complete finite-volume hyperbolic 3-manifold $N$, start with either a slightly shrunken maximal horotorus neighborhood $V$ of a cusp or slightly shrunken maximal tube $\mathrm{V}$ about a geodesic. After expanding $V$ in the normal direction, it eventually encounters itself, thereby creating a 1-handle. Subsequent expansions give rise to the creation of 1, 2 , and 3-handles. In the presence of low volume we expect that $V$ will rapidly encounter 1 and 2-handles and $\partial V$ together with a subset of these handles (perhaps somewhat perturbed to allow for the "valence-3 2-handle condition") will create a Mom- $n$ manifold $M$, for some $n \leq 4$. Furthermore, the complement of $M$ will consist of cusp neighborhoods and tubular neighborhoods of geodesics. In practice, the handle structure may arise in a somewhat different manner; e.g., as a sub-complex of the dual triangulation of the Ford domain (see [GMM3]).

The papers [GM] and [GMM] can be viewed as steps in this direction when $V$ is a tubular neighborhood about a geodesic $\gamma$. Indeed, [GM] gives a lower bound on $\operatorname{Vol}(N)$ in terms of the tube radius of $\gamma$ and [GMM] gives a lower bound in terms of the first two ortholengths, or equivalently the radii of the expanding $V$ as it encounters its first and second 1-handles.

Definition 0.6. If $i: M \rightarrow N$ is an embedding, then we say that the embedding is elementary if $i_{*} \pi_{1}(M)$ is Abelian, and non-elementary otherwise. When the context is clear, we will refer to embedded submanifolds of $N$ as either elementary or nonelementary. In this paper, $N$ will be a compactified cusped hyperbolic 3-manifold of finite volume and $M$ is a compact 3-manifold embedded in the interior of $N$.

The strength of our method derives from three things. First, our definition of Mom- $n$ manifolds appears to be the correct definition for attacking questions about low-volume hyperbolic 3-manifolds (we give the basic definitions in this introduction and in $\S 1$, and set down some basic facts about handle structures in §2). Second, from the Mom- $n$ definition we are able to construct a bridge between topology and geometry. This is achieved in $\S 3$ and $\S 4$, where we prove that if $n \leq 4$, then given a non-elementarily embedded Mom- $n$ manifold $M_{1}$ in a cusped hyperbolic manifold $N$ we can find a non-elementarily embedded compactified cusped hyperbolic 
Mom- $k$ manifold $M_{2}$ in $N$, where $k \leq n$, with weaker results for general values of $n$. Third, we provide a powerful tool for exploiting this topological-geometric bridge by classifying all hyperbolic Mom-2 and Mom-3 manifolds, and conjecturally classifying Mom-4 manifolds. This classification is carried out in $\$ 5$ where we prove Theorem 5.1 and state Conjecture 5.3, which we summarize here as follows:

Theorem. There are 3 hyperbolic Mom-2 manifolds and 21 hyperbolic Mom-3 manifolds (including the 3 hyperbolic Mom-2's, which are also Mom-3's).

Conjecture. There are 138 hyperbolic Mom-4 manifolds (including the hyperbolic Mom-2's and Mom-3's, which are also Mom-4's).

In $\S 6$ we clean up a loose end by proving that any non-elementary embedding of a hyperbolic Mom- $n$ manifold $M, n \leq 4$, into a compact hyperbolic manifold $N$ gives rise to an internal Mom- $n$ structure on $N$, i.e., every component of $\partial M$ either splits off a cusp of $\stackrel{\circ}{N}$ or bounds a solid torus in $N$. In either case it is to the outside of $M$.

Definition 0.7. The Mom number of the compact hyperbolic 3-manifold $N$ is the smallest $n$ such that its interior is obtained from a Mom- $n$ manifold by capping off the toral boundary components with solid tori and cusps.

We believe that a reasonable solution to Conjecture 0.1 will be given by a solution to the following

Conjecture 0.8. Low volume compact hyperbolic 3-manifolds have low Mom number.

In $\$ 7$ we give examples of internal Mom-2 structures on cusped hyperbolic 3manifolds, including in particular a detailed exposition of one of our key motivating examples.

The authors wish to thank Morwen Thistlethwaite for his assistance in identifying the hyperbolic manifolds in $\S 5$.

\section{Basic definitions and lemmas}

Notation 1.1. If $X$ is a space, then let $|X|$ denote the number of components of $X$, $\stackrel{\circ}{X}$ denote the interior of $X$ and $N(X)$ denote a regular neighborhood of $X$ in some ambient space in which it is embedded. Similarly, $\bar{X}$ denotes the closure of $X$.

Definition 1.2. Let $M$ be a compact connected 3-manifold $M$ with $B \subset \partial M$ a compact surface which may be either disconnected or empty. A handle structure $\Delta$ 
on $(M, B)$ is the structure obtained by starting with $B \times I$, adding a finite union of 0 -handles, then attaching finitely many 1 and 2-handles to $B \times\{1\}$ and the 0 -handles. We call $B \times I$ (resp. $B \times I \cup 0$-handles) the base (resp. extended base) and say that the handle structure is based on $B$.

Most of the time $B$ will be a component of $\partial M$. We strongly recommend that the reader think only about this case until absolutely necessary. Sometimes, $B$ will be an $\pi_{1}$-injective annulus in $\partial M$. In that case, $M$ will be a manifold with corners, the corners being $\partial B \times\{0,1\}$; see the manifold $M_{1}$ in Example 7.1 for a typical case of this. In the most general case (in this paper), $B$ will have several components, each being either an annulus or torus as above.

In this paper all $k$-handles will attach to lower dimensional handles in a standard way. E.g. if a 1-handle is parametrized by $D^{2} \times I$, then $D^{2} \times \partial I$ is the attaching zone and a 2-handle will attach to the 1-handle in regions of the form $\alpha \times I$, where $\alpha$ is an embedded arc in $\partial D^{2}$. For a precise statement see Definition 2.1.

The valence of a 1-handle is the number of times, counted with multiplicity, the various 2-handles run over it and the valence of a 2-handle is the number of 1-handles, counted with multiplicity, it runs over.

Following the terminology of Schubert [Sch] and Matveev [Mv1] we call the 0-handles, 1-handles and 2-handles balls, beams and plates respectively. We call islands (resp. bridges) the intersection of the extended base with beams (resp. plates) and the components of the closure of the complement of the islands and bridges in $B \times\{1\} \cup \partial(0$-handles $)$ are the lakes. We say that $\Delta$ is full if each lake is a disc. If $B=\emptyset$, then we say that $\Delta$ is a classical handle structure.

Let $M$ be a compact 3-manifold with $\partial M$ a union of tori and let $T$ be a component of $\partial M$. We say that $(M, T, \Delta)$ is a weak Mom- $n$ if $\Delta$ is a handle structure based on $T$ without 0 -handles or 3-handles, such that each 1-handle is of valence $\geq 2$ and each 2-handle is of valence 2 or 3. Furthermore, there are exactly $n$ 2-handles of valence 3 . A weak Mom- $n$ with no valence-2 2-handles is a Mom- $n$. A weak Mom- $n$ is strictly weak if there exists a valence-2 2-handle.

Remark 1.3. For Euler characteristic reasons, if $(M, T, \Delta)$ is a weak Mom- $n$, then $\Delta$ has the same number of 1 and 2-handles.

The following is a well-known existence result stated in our language.

Proposition 1.4. A compact 3-manifold $M$ has a weak Mom-structure if and only if $\partial M$ is a union of at least two tori.

Proof. If $M$ has a weak Mom- $n$ structure, then by definition all of its boundary components are tori and there is at least one such boundary component. Further, because there are no 3-handles in $\Delta$, there must be another (torus) boundary component. 
The converse is not much more difficult. In fact, if $M$ has at least two boundary components, then it is standard to create it by first starting with a thickened boundary component and then adding 1 and 2-handles where the 1 and 2-handles are of valence $\geq 2$. By subdividing the 2-handles with 1-handles we satisfy the condition that the 2-handles have valence $\leq 3$.

Definition 1.5. Call a torus that bounds a solid torus a tube and call a torus bounding a tube with knotted hole a convolutube. Recall that a tube with knotted hole is a $B^{3}-\stackrel{\circ}{N}(\gamma)$, where $\gamma$ is a knotted proper arc.

The following standard result follows from the loop theorem (see e.g. [Jac]).

Lemma 1.6. If $S$ is a torus in an irreducible 3-manifold $N$, then either $S$ is incompressible or $S$ is a tube or a convolutube. If $S \subset N, \partial N$ is incompressible and $S$ is compressible, and there exists an embedded essential annulus connecting $S$ to a component of $\partial N$, then $S$ is a tube.

Proposition 1.7. If $M$ is a non-elementary compact, connected 3-manifold embedded in the compact hyperbolic 3-manifold $N$ and $\partial M$ is a union of tori, then, up to isotopy, $N$ is obtained from $M$ by first filling a subset of the components of $\partial M$ by solid tori to obtain the manifold $M_{1}$, then compressing a subset of the components of $\partial M_{1}$ to obtain the manifold $M_{2}$, then attaching 3-balls to the 2-sphere components of $\partial \mathrm{M}_{2}$ to obtain $M_{3}$. Furthermore all of these operations can be performed within $N$.

Proof. The components of $\partial M$ that bound solid tori in $N$ are exactly those boundary components which compress to the non- $M$ side. Fill in all such tori to obtain the manifold $M_{1}$. If $P$ is a component of $\partial M_{1}$ which is not boundary parallel in $N$, then $P$ is compressible in $N$ and hence is a convolutube. These convolutubes can be isotoped to lie in pairwise disjoint 3-balls in $N$. Therefore we can compress all the compressible components of $\partial M_{1}$ (to obtain $M_{2}$ ) and cap the resulting 2-spheres with 3-cells to obtain $M_{3}$ which is isotopic to $N$.

Since $M_{3}$ must have all boundary components boundary parallel in $N$ and $M_{3}$ is non-elementary, the result follows.

Corollary 1.8. Let $M \subset \stackrel{\circ}{N}$ be a connected compact non-elementary submanifold in the compact hyperbolic 3-manifold $N$. If $\partial M$ is a union of tori, then each component of $\partial N$ is parallel to a component of $\partial M$ via a parallelism disjoint from $M$.

The following result is due to Kerckhoff (see [Koj]).

Lemma 1.9. If $\gamma$ is a simple closed geodesic in the complete, finite-volume hyperbolic 3-manifold $N$, then $N-\gamma$ has a complete finite-volume hyperbolic structure. 
Lemma 1.10. Let $M$ be a compact connected non-elementary submanifold of the compact hyperbolic 3-manifold $N$ such that $\partial M$ is a union of tori.

i) If $M_{1}=\overline{M-V}$, where $V$ is a solid torus or 3-ball with $\partial V \subset M$, or $M_{1}$ is obtained by deleting an open regular neighborhood of a properly embedded arc from $M$, then $M_{1}$ is non-elementary.

ii) If $M^{\prime}$ is obtained by compressing $\partial M$, then a unique component $M_{1}$ of $M^{\prime}$ is non-elementary.

iii) If $A \subset M$ is a properly embedded annulus in $M$, then a unique component $M_{1}$ of the manifold $M^{\prime}$ obtained by splitting $M$ along $A$ is non-elementary.

iv) If $F \subset \stackrel{\circ}{M}$ is an embedded torus, then a unique component $M_{1}$ of the manifold $M^{\prime}$ obtained by splitting $M$ along $F$ is non-elementary.

Proof. i) Both $M_{1}$ and $M$ have the same $\pi_{1}$-image.

ii) Let $S$ denote the 2-sphere component of $\partial M^{\prime}$ and $B$ the 3-ball bounded by $S$. If $M^{\prime}$ is connected, then $B \cap \stackrel{\circ}{M^{\prime}}=\emptyset$, or else $\pi_{1}(M)$ is cyclic. It follows that $M$ and $M^{\prime}$ have the same $\pi_{1}$-image. If $M^{\prime}$ is disconnected, then some component $M_{2}$ lies in $B$ hence has trivial $\pi_{1}$-image in $N$ and hence the other component $M_{1}$ has the same $\pi_{1}$-image as $M$.

iii) The boundary of each component of $M$ split along $A$ is also a union of tori. We consider the case where the split manifold $M^{\prime}$ is connected, for the general case is similar. Since all tori in $N$ separate, $M$ is obtained from $M^{\prime}$ by attaching a thickened annulus $A$ to a boundary parallel torus, a tube or a convolutube $T$.

If $T$ is boundary parallel and $A$ attaches to the cusp side of $T$, then $M^{\prime}$ and $M$ have the same $\pi_{1}$-image. If $A$ attaches to the non-cusp side, then it must attach along essential curves in $T$, else some component of $\partial M$ is a 2 -sphere. Since $N$ is hyperbolic, $A$ is boundary parallel. It follows that $M$ is elementary, a contradiction.

If $T$ is a tube, let $V$ denote the solid torus bounded by $T$. If $A \subset V$ then $M$ and $M^{\prime}$ have the same $\pi_{1}$-image. If $A$ attaches to the outside of $V$, then let $X=V \cup N(A)$. Note that $X$ and $M$ have the same $\pi_{1}$-image. If some component of $\partial A$ was inessential in $\partial V$, then either $\partial M$ contains a 2 -sphere or the $\pi_{1}$-image of $X$ is cyclic. If some component of $\partial A$ bounded a disc in $V$, then again $X$ has cyclic $\pi_{1}$-image, since $N$ is irreducible. In the remaining case, $X$ is a Seifert fibered space with incompressible boundary. It is well known that there is no non-elementary embedding of such a space into a hyperbolic 3-manifold, e.g. see [JS], [Jo] for related material.

If $T$ is a convolutube, and $A$ attaches to the inside, then $M^{\prime}$ has the same $\pi_{1}$ image as $M$. If $A$ attaches to the outside, then $M$ has cyclic $\pi_{1}$-image.

iv) Again $F$ is either boundary parallel or a tube or a convolutube. In each case one component $M_{1}$ of $M$ split along $F$ has the same $\pi_{1}$-image as $M$ while the other component has Abelian $\pi_{1}$-image. 
Lemma 1.11. If $M$ is compact, irreducible, and non-elementary, and if $\partial M$ is a union of tori, then $\partial M$ is incompressible.

Proof. If $M^{\prime}$ is obtained by compressing $\partial M$, then $\partial M^{\prime}$ has a 2-sphere component $S$ that bounds a 3-ball $B$ in $M$ and hence in $M^{\prime}$. It follows that either the compression is inessential or $M$ is a solid torus. The latter implies that $M$ is elementary.

Definition 1.12. Let $N$ be a compact hyperbolic 3-manifold. An internal Mom-n structure on $N$ consists of a non-elementary embedding $f: M \rightarrow N$ where $(M, T, \Delta)$ is a Mom- $n$ and each component of $\partial M$ is either boundary parallel in $N$ or bounds a solid torus in $N$. We will sometimes suppress mention of the embedding and simply say that $(M, T, \Delta)$ is an internal Mom- $n$ structure on $N$. In the natural way we define the notion of weak internal Mom-n structure on $N$.

Lemma 1.13. A non-elementary embedding of the Mom- $n$ manifold $M$ into the compact hyperbolic 3-manifold $N$ will fail to give an internal Mom-n structure on $N$ if and only if some component of $\partial M$ maps to a convolutube. In that case, a reimbedding of $M$, supported in a neighborhood of the convolutubes gives rise to an internal Mom-n structure on $N$.

Proof. As in the proof of Lemma 1.7, there exists a finite set of pairwise disjoint embedded 3-balls such that each convolutube is contained in exactly one such ball. Reimbed $M$ in $N$ by unknotting each convolutube. The boundary components of the resulting reimbedded $M$ will be either tubes or essential annuli. Further the reimbedded $M$ will have the same $\pi_{1}$-image as $M$.

Given a Mom- $n$ structure $(M, T, \Delta)$ in a hyperbolic manifold $N$, we very much want $\Delta$ to be full. A common problem with non-full structures in what follows is that if $\Delta$ contains a lake which is a $\pi_{1}$-injective annulus in $T \times\{1\}$, then $M$ clearly contains an essential annulus and hence is not hyperbolic. We will frequently try to fix this problem by splitting $M$ open along the essential annulus to obtain a new manifold $M_{1}$, with one fewer torus boundary components. However the handle structure $\Delta_{1}$ that $M_{1}$ inherits from $M$ is no longer based on a torus, but rather is based on the annulus that remains when $T$ is split. This necessitates the following definition:

Definition 1.14. A general based Mom- $n(M, B, \Delta)$ consists of a compact 3-manifold $M$ with $\partial M$ a union of tori, $B \subset \partial M$ a compact codimension- 0 submanifold of $\partial M$ that is $\pi_{1}$-injective in $\partial M$, and $\Delta$ a handle structure for $M$ based on $B$ without 0 handles such that every 1 -handle is of valence $\geq 2$, every 2-handle is of valence 3 and there are exactly $n$ of each of them. A weak general based Mom- $n$ is as above with $\Delta$ having $k \geq 0$ extra valence-2 2-handles. Note that such a $\Delta$ has $k+n$ 1-handles. 
A general based internal Mom-n structure on $N$ consists of a non-elementary embedding $f: M \rightarrow N$, where $N$ is a compact hyperbolic 3-manifold, no component of $\partial M$ is a convolutube and $(M, B, \Delta)$ is a general based Mom- $n$ structure. Along similar lines we have the notion of weak general based internal Mom- $n$ structure on $N$.

Remark 1.15. See the second part of Example 7.1 for a simple non-trivial example of a general based Mom-2 structure arising from a non-full Mom-2 structure on the figure-8 knot complement. Whenever we obtain a general based Mom- $n$ structure, our wish is to transform it into a "proper" Mom- $k$ structure $(M, T, \Delta)$ with $k \leq n$, but it is questionable whether or not we can always do this for $n>4$; see also Problem 2.13.

Notation 1.16. If $\sigma$ is a 2-handle (resp. $\eta$ is a 1-handle), then let $\delta(\sigma)(\operatorname{resp} . \delta(\eta))$ denote the lateral boundary, i.e., the closure of that part of $\partial \sigma$ (resp. $\partial \eta$ ) which does not lie in lower index handles. If $b$ is a bridge which lies in the 2-handle $\sigma$, then define $\delta(b)=b \cap \delta \sigma$.

\section{Handle structures and normal surfaces}

We slightly modify Haken's [Ha] theory of surfaces in handlebodies to our setting. We closely parallel the excellent exposition given by Matveev in [Mv1].

Definition 2.1. Let $\Delta$ be a handle structure on $M$ based on $B \subset \partial M$. A compact surface $F \subset M$ is called normal if the following holds:

(1) $F$ intersects each plate $D^{2} \times I$ in parallel copies of the form $D^{2} \times\{\mathrm{pt}\} \subset$ $D^{2} \times(0,1)$.

(2) Each component of the intersection of $F$ with a beam $D^{2} \times I$ is of the form $\alpha \times I$, up to isotopy, where $\alpha$ is a proper arc whose endpoints are disjoint from $\delta$ (bridges). Furthermore, each component of $D^{2} \times\{0\}-\alpha$ intersects $\delta$ (bridges) in at least two points. (See the Remarks 2.2.)

(3) Each component $U$ of $F \cap(B \times[0,1] \cup 0$-handles $)$ is $\pi_{1}$-injective in $B \times$ $[0,1] \cup 0$-handles. If $U \cap B \times\{0\} \neq \emptyset$, then $U$ is a product disc or product annulus, i.e., the inclusion $(U, U \cap(B \times\{0\}), U \cap(B \times\{1\}), U \cap(\partial B \times I)) \rightarrow$ $(B \times I, B \times\{0\}, B \times\{1\}, \partial B \times I)$ can be relatively isotoped to a vertical embedding of $\alpha \times I$ into $B \times I$, where $\alpha \times\{0\}$ is either an essential simple closed curve or an essential proper arc in $B \times\{0\}$.

Remarks 2.2. i) For $F$ closed, the second condition can be restated by requiring that $\alpha$ intersect distinct components of $D^{2} \times\{0\} \cap$ (bridges). When $\partial F \neq \emptyset$, the second 
condition implies that $F$ is locally efficient in that it neither can be locally boundary compressed nor can its weight be reduced via an isotopy supported in the union of a 2-handle and its neighboring 1-handles.

ii) Note that $\partial F$ lies in the union of the beams, lakes and $B \times\{0\}$.

Lemma 2.3 (Haken). If $F$ is a compact, incompressible, boundary-incompressible, properly embedded surface in a compact irreducible 3-manifold, then $F$ is isotopic to a normal surface.

Definition 2.4. Let $\Delta$ be a handle structure on $M$ based on $B \subset \partial M$. The valence $v(b)$ of a beam (resp. plate) is the number of plates (resp. beams) that attach to it, counted with multiplicity. Define the complexity $C(\Delta)$ to be $\left(\rho_{1}(\Delta),\left|\Delta^{1}\right|,\left|\Delta^{2}\right|\right)$ lexicographically ordered, where $\rho_{1}(\Delta)=\Sigma_{\text {beams } b} \max (v(b)-2,0)$ and $\left|\Delta^{i}\right|$ is the number of $i$-handles for $i=1,2$. In particular we have the

$\rho_{1}$-formula:

$\rho_{1}(\Delta)=\sum_{\text {2-handles } \sigma} v(\sigma)-2\left|\Delta^{1}\right|+\mid\{$ valence-1 1-handles $\}|+2|\{$ valence-0 1-handles $\} \mid$.

Lemma 2.5 (Matveev). Let $\Delta$ be a handle structure on $M$ based on $B, F \subset M$ a closed normal surface and let $M^{\prime}$ be $M$ split along $F$. If each component of $M^{\prime} \cap B \times[0,1]$ disjoint from $B \times\{0\}$ is a 3-ball, then $M^{\prime}$ has a handle structure $\Delta^{\prime}$ based on $B$ with $\rho_{1}\left(\Delta^{\prime}\right)=\rho_{1}(\Delta)$.

Proof. This follows almost exactly as in $\S 3$ and $\S 4$ of [Mv1]: $M^{\prime}$ naturally inherits a handle structure $\Delta_{1}$ from $\Delta$ as follows. The surface $F$ splits $B \times I$ into various submanifolds one of which is homeomorphic to $B \times[0,1]$ with $B \times\{0\}=B$. All of the other submanifolds which lie in $M^{\prime}$ are 3-balls. This new $B \times[0,1]$ becomes the base and the 3-balls become 0 -handles. The various 1 and 2-handles are split by $F$ into 1 and 2-handles and as in [Mv1], $\rho_{1}\left(\Delta_{1}\right)=\rho_{1}(\Delta)$.

Lemma 2.6. Given the handle structure $\Delta$ on $(M, B)$, if some 1-handle is valence-1, then there exists another structure $\Delta_{1}$ on $(M, B)$ with $C\left(\Delta_{1}\right)<C(\Delta)$.

Proof. Cancel the valence-1 1-handle with the 2-handle that it hits to obtain $\Delta_{1}$. Note that $\rho_{1}\left(\Delta_{1}\right)=\rho_{1}(\Delta)$ and $\left|\Delta_{1}^{1}\right|=\left|\Delta^{1}\right|-1$.

Lemma 2.7. If $(M, T, \Delta)$ is a Mom-n structure then $C(\Delta)=(n, n)$.

Lemma 2.8. Let $\Delta$ be a handle structure on $(M, B), F \subset M$ a connected separating normal surface disjoint from $B$ and let $M_{1}$ be the component of $M-\stackrel{\circ}{N}(F)$ which does not contain $B$. If each component of $F \cap B \times[0,1]$ is a disc, then $M_{1}$ has a classical handle structure $\Delta_{1}$ with $\rho_{1}\left(\Delta_{1}\right) \leq \rho_{1}(\Delta)$. 
Proof. This follows as in the proof of Lemma 2.5 after noting that each component of $M_{1} \cap(B \times[0,1])$ is a 3-ball and these 3-balls correspond to the 0 -handles of the induced handle structure on $M_{1}$.

Lemma 2.9. If $\partial M$ is a union of tori, and $\Delta$ is a handle structure on $(M, T)$ with $T$ a component of $\partial M$, then there exists a weak Mom- $n\left(M, T, \Delta_{1}\right)$ with $n \leq \rho_{1}\left(\Delta_{1}\right)$.

Proof. First apply Lemma 2.6, then add 1-handles to subdivide the valence- $k, k \geq 4$, 2-handles into valence-3 2-handles.

Definition 2.10. In our setting the well known operation of turning a handle structure upside down transforms a handle structure $\Delta$ on $(M, T)$ to a handle structure $\Delta_{1}$ on $(M, \overline{(\partial M \backslash T)})$ where $i$-handles of $\Delta$ become $3-i$-handles of $\Delta_{1}$.

Lemma 2.11. If $\Delta_{1}$ is obtained by turning $\Delta_{0}$ upside down and the valence of each 1 and 2-handle of $\Delta_{0}$ is at least 2 , then $\rho_{1}\left(\Delta_{0}\right)=\rho_{1}\left(\Delta_{1}\right)$.

Definition 2.12. If $B \neq \emptyset$ is a compact $\pi_{1}$-injective submanifold of $\partial M$, then define $\operatorname{rank}_{\rho_{1}}(M, B)$ to be the least $n$ such that there exists a handle decomposition $\Delta$ on $(M, B)$ with $\rho_{1}(\Delta)=n$.

Problem 2.13. Is there an example of a compact hyperbolic 3-manifold $N$ with $T$ a component of $\partial N$ and $A$ an essential annulus in $T$ such that $\operatorname{rank}_{\rho_{1}}(N, A)<$ $\operatorname{rank}_{\rho_{1}}(N, T) ?$

\section{Estimates for the reduction of $\rho_{1}$ under splitting}

The main result of these next two sections is Theorem 4.1 which shows that if a compact hyperbolic 3-manifold $N$ (i.e., a compactified cusped hyperbolic 3-manifold) has an internal Mom- $n$ structure $(M, T, \Delta)$ with $\Delta$ full and $n \leq 4$, then it has an internal Mom- $k$ structure $\left(M_{1}, T_{1}, \Delta_{1}\right)$ where $k \leq n, \Delta_{1}$ is full, and $M_{1}$ is compact hyperbolic. If $n>4$, we obtain the similar conclusion except that "full" is replaced by "general based" where $T_{1}$ is a union of tori and annuli.

As far as we know, transforming a structure based on an annulus lying in a component $U$ of $\partial M$ to one based on the whole torus $U$ may require an increase in $\rho_{1}$. This issue is responsible for many of the technicalities of this section and the next. See Problem 2.13.

Definition 3.1. Let $(M, B, \Delta)$ be a handle structure with a 1-handle $\eta$ that attaches to the 0 -handle $V$ at each end. We say that $\left(M_{1}, B_{1}, \Delta_{1}\right)$ is obtained by hollowing out $V$ and $\eta$ if $M_{1}$ is obtained by deleting the interior of $V$ (except for a very thin 
regular neighborhood of $\partial V \backslash \eta$ ) as well as a neighborhood of the core of $\eta$. Here $\Delta_{1}$ is based on $B_{1}$, where $B_{1}$ is the union of $B$ and the newly created torus component of $\partial M_{1}$.

Lemma 3.2. Let $M$ be non-elementarily embedded in $N$ with handle structure $(M, B, \Delta)$. If $\left(M_{1}, B_{1}, \Delta_{1}\right)$ is obtained by hollowing out the 0-handle $V$ and the 1 -handle $\eta$, then $M_{1}$ is non-elementarily embedded and $\rho_{1}\left(\Delta_{1}\right)=\rho_{1}(\Delta)-\max (v-$ $2,0)$ where $v$ is the valence of $\eta$.

Proof. That $M_{1}$ is non-elementary follows from Lemma 1.10 while the fact about $\rho_{1}$ is immediate.

Lemma 3.3. Let $M$ be a non-elementary 3-manifold in the compact hyperbolic 3manifold $N$ such that $\partial M$ is a union of tori and let $(M, B, \Delta)$ be a handle structure. If there exists either a lake of $\Delta$ that is compressible in $B \times I$ or a valence-0 2-handle, then there exists a handle structure $\left(M^{\prime}, B^{\prime}, \Delta^{\prime}\right)$ where $M^{\prime}$ is non-elementary, $\partial M^{\prime}$ is a union of tori, and $C\left(\Delta^{\prime}\right)<C(\Delta)$.

Proof. If $\Delta$ has a compressible lake, then let $\left(M_{1}, B, \Delta_{1}\right)$ be obtained by compressing the lake along the disc $D$. Note that the 3-ball $E$ bounded by $D$ and a disc in $B \times\{1\}$ is a 0 -handle of $\Delta_{1}$. Some component $M_{2}$ of $M_{1}$ is non-elementary by Lemma 1.10. Let $\left(M_{2}, B_{2}, \Delta_{2}\right)$ be the induced handle structure. If some component of $\partial M_{2}$ is a 2-sphere $S$, then let $\left(M_{3}, B_{3}, \Delta_{3}\right)$ be obtained by deleting from $\Delta_{2}$ a 2-handle whose lateral boundary intersects $S$. If $B_{3} \neq \emptyset$, then let $\left(M^{\prime}, B^{\prime}, \Delta^{\prime}\right)=\left(M_{3}, B_{3}, \Delta_{3}\right)$, otherwise obtain $\left(M^{\prime}, B^{\prime}, \Delta^{\prime}\right)$ by hollowing out the 0-handle $E$ and some 1-handle of $\Delta_{3}$.

Now assume that $\Delta$ has no compressible lakes. If $\Delta$ has a valence-0 2-handle $\sigma$, then the attaching curve is essential in $B \times\{1\}$. Let $\left(M_{1}, B_{1}, \Delta_{1}\right)$ be the handle structure obtained by compressing $M$ along an extended core $D$ of $\sigma$ and absorbing the split 2-handle into the base. Note that either $B_{1}$ contains a 2-sphere component $S$ or has two $D^{2}$ components depending whether or not the component hit by $D$ is a torus or annulus. In former case $M_{1}$ is non-elementary (by Lemma 1.10) and $S$ bounds a 3-ball $V$ to the outside of $M_{1}$. Let $\left(M_{2}, B_{2}, \Delta_{2}\right)$ be obtained by adding $V$ to $M_{1}$. If $B_{2}=\emptyset$, then $\Delta_{2}$ is a classical handle structure with a unique 0 -handle. Finally hollow out the 0 -handle and a 1-handle to obtain the desired $\left(M^{\prime}, B^{\prime}, \Delta^{\prime}\right)$.

If $B_{1}$ has two $D^{2} \times I$ components, then transform them into 0-handles. Let $M_{2}$ be the non-elementary component of $M_{1}$ and $\left(M_{2}, B_{2}, \Delta_{2}\right)$ the induced handle structure. If some components of $B_{2}$ are inessential annuli in $\partial M_{2}$, then enlarge the 0 -handles to absorb them. Now complete the proof as in the previous paragraph.

Remark 3.4. From now on, unless otherwise stated, we will assume that if a homotopically inessential lake or valence-0 2-handle appears, then it is immediately removed as in the proof of the above lemma. 
Lemma 3.5. Let $M$ be a non-elementarily embedded 3-manifold in the compact hyperbolic 3-manifold $N$ and $\partial M$ a union of tori. If $\Delta$ is a general based handle structure on $(M, B)$ (with possibly $B=\emptyset)$, then $\rho_{1}(\Delta)>0$.

Proof. By canceling 0-handles with 1-handles we can assume that there exist no 0handles, unless $B=\emptyset$, in which case we can assume that $\Delta$ has a unique 0 -handle. In that case, hollow out the 0 -handle and a 1 -handle to reduce to the case $B \neq \emptyset$. Since these operations are not $\rho_{1}$-increasing, it suffices to prove the lemma in this case. By further canceling we can assume that there are no valence-1 1-handles. If $\rho_{1}(\Delta)=0$, then all 1-handles are of valence- 2 and so $M$ is obtained by gluing an $I$-bundle $J$ to $B \times I$ where the vertical boundary of $J$ is glued to annuli in $B \times\{1\}$. By Lemma 3.3, we can assume that no component of $J$ is a $D^{2} \times I$ and hence each component has non-positive Euler characteristic. Since $\chi(M)=\chi(B)=0$, it follows that each component of $J$ has zero Euler characteristic, hence is an $I$-bundle over an annulus or Möbius band. It follows from Lemma 1.10 that $M$ is elementary, a contradiction.

The following very crude warm up lemma roughly asserts that any handle structure $(M, B, \Delta)$ on a non-elementary manifold whose boundary is a union of tori can be transformed to a weak general based internal Mom-k structure $\left(M^{\prime}, B^{\prime}, \Delta^{\prime}\right)$ without increasing complexity. If $\Delta$ was not general based, then neither is $\Delta^{\prime}$. Also if we insist that $M^{\prime}$ be irreducible then $\rho_{1}$ is non-increasing. Subsequent arguments will show that under stronger hypotheses on $\Delta$ these transformations lead to reductions in $\rho_{1}$.

Lemma 3.6. Let $M$ be non-elementarily embedded in the compact hyperbolic 3manifold $N$ with $\partial M$ a union of tori and let $(M, B, \Delta)$ be a general based handle structure. Then there exists a weak general based internal Mom- $k$ structure $\left(M^{\prime}, B^{\prime}, \Delta^{\prime}\right)$, with $M^{\prime}$ non-elementary, $\partial M^{\prime}$ a union of tori and $C\left(\Delta^{\prime}\right) \leq C(\Delta)$. If $B$ is a torus (i.e., $\Delta$ is not general based), then so is $B^{\prime}$. If $M$ is hyperbolic, then either $M^{\prime}=M$ or $\rho_{1}\left(\Delta^{\prime}\right)<\rho_{1}(\Delta)$.

If $M$ is reducible, then there exists a $\left(M^{\prime}, B^{\prime}, \Delta^{\prime}\right)$ with $M^{\prime}$ irreducible, nonelementary, $\partial M^{\prime}$ a union of tori and $\rho_{1}\left(\Delta^{\prime}\right) \leq \rho_{1}(\Delta)$.

Proof. By Lemma 3.3 we can assume that every 1 and 2 -handle has valence $\geq 1$. It is routine to cancel the 1 and 2-handles of valence 1 without increasing complexity; then note by Lemma 3.5 there must exist at least one 1 -handle of valence $\geq 3$. If $M$ is hyperbolic, $\partial M$ is incompressible in $M$ so these operations do not change $M$. If $B=\emptyset$, then $\Delta$ is classical and we can assume that it has a single 0 -handle. By hollowing out this 0 -handle and a valence $\geq 31$-handle we obtain a handle structure $\left(M_{1}, T, \Delta_{1}\right)$ where $M_{1}$ is non-elementary, $T$ is the newly created component of $\partial M_{1}$ and $\rho_{1}\left(\Delta_{1}\right)<\rho_{1}(\Delta)$. 
If $M$ is reducible, then let $F$ be a normal reducing essential 2-sphere and let $\left(M_{1}, B_{1}, \Delta_{1}\right)$ be the handle structure obtained by splitting along $F$. Let $M_{2}$ be the non-elementary component of $M_{1}, B_{2}=B_{1} \cap M_{2}$ and $\Delta_{2}$ the restriction of $\Delta_{1}$ to $M_{2}$. Note that $\rho_{1}\left(\Delta_{2}\right) \leq \rho_{1}(\Delta)$.

If possible obtain $\left(M_{3}, B_{3}, \Delta_{3}\right)$ by deleting a 2-handle whose lateral boundary intersects the 2-sphere component $S$ of $\partial M_{2}$ in exactly one component. Observe that $\left|\partial M_{3}\right|<|\partial M|$ and $\rho_{1}\left(\Delta_{3}\right) \leq \rho_{1}(\Delta)$. If every 2-handle with lateral surface intersecting $S$ has both its lateral surfaces intersecting $S$, then since $M$ is connected each lateral surface of every 2 -handle intersects $S$. Obtain $\left(M_{3}, B_{3}, \Delta_{3}\right)$ by deleting a 2-handle that attaches to a valence $\geq 31$-handle. Observe that $\rho_{1}\left(\Delta_{3}\right)<\rho_{1}(\Delta)$ and $\left|\partial M_{3}\right| \leq|\partial M|$. After finitely many such operations we obtain an irreducible 3-manifold $M^{\prime}$. Since $M^{\prime}$ is non-elementary and its boundary is a union of tori, it has incompressible boundary. Now complete the proof as in the first paragraph.

Similarly we have

Lemma 3.7. Let $M$ be non-elementarily embedded in the compact hyperbolic 3manifold $N$ with $\partial M$ a union of tori and let $(M, \Delta)$ be a classical handle structure. Then there exists a classical handle structure $\left(M^{\prime}, \Delta^{\prime}\right)$ such that $M^{\prime}$ is nonelementary, $\partial M^{\prime}$ is a union of tori, $\rho_{1}\left(\Delta^{\prime}\right) \leq \rho_{1}(\Delta), \Delta^{\prime}$ has a unique 0 -handle, and every 1 and 2-handle has valence $\geq 2$.

Lemma 3.8. Let $(M, B, \Delta)$ be a handle structure such that each 1-handle is of valence $\geq 3$ and $B$ contains an annulus component A. Suppose there exists an essential arc $\sigma \times\{1\}$ in $A \times\{1\}$ disjoint from the 1-handles crossing the cores of 2handles exactly once, transversely. Then there exists a handle structure $\left(M, B^{\prime}, \Delta^{\prime}\right)$ such that $\rho_{1}\left(\Delta^{\prime}\right)<\rho_{1}(\Delta)$.

Proof. Replace $A \times I$ by a 0 -handle and 1-handle to create a handle structure $\Delta_{1}$, possibly classical, based on $B \backslash A$. More precisely, if $A=X \cup N(\sigma)$ where $X$ is the closure of $A \backslash N(\sigma)$, then the 0 -handle is $X \times I$ and the 1-handle is $N(\sigma) \times I$. Here $N(\sigma)$ is sufficiently small so that all the 1-handles of $\Delta$ which attached to $A \times\{1\}$ now attach to the 0 -handle. Thus all the handles of $\Delta$ can be viewed as handles of $\Delta_{1}$ and so $\rho_{1}\left(\Delta_{1}\right)=\rho_{1}(\Delta)$. The 1-handle of $\Delta_{1}$ corresponding to $N(\sigma) \times I$ is of valence 1 while all the others are of valence at least three. Let $\Delta_{2}$ be the handle structure obtained by canceling this 1 -handle with its 2 -handle. Then $\rho_{1}\left(\Delta_{2}\right)<\rho_{1}\left(\Delta_{1}\right)$.

Lemma 3.9. Let $f: M \rightarrow N$ be a non-elementary embedding of a compact connected 3-manifold into a compact irreducible 3-manifold. Suppose $\partial M$ is a union of tori, $T$ is a component of $\partial M$, and $\Delta$ is a handle structure on $(M, T)$ without 0 -handles such that each 2-handle is of valence $\geq 3$. If there exists a valence-0 1-handle (resp. two valence-1 1-handles, resp. one valence-1 1-handle) then there 
exists a non-elementary embedding $g: M^{\prime} \rightarrow N$ with $\partial M^{\prime}$ a union of tori and a handle structure $\Delta^{\prime}$ on $\left(M^{\prime}, T^{\prime}\right)$ with $T^{\prime}=T$ such that $\rho_{1}\left(\Delta^{\prime}\right) \leq \rho_{1}(\Delta)-3$ (resp. $\rho_{1}\left(\Delta^{\prime}\right) \leq \rho_{1}(\Delta)-3$, resp. $\rho_{1}\left(\Delta^{\prime}\right) \leq \rho_{1}(\Delta)-2$ ) and $\Delta^{\prime}$ contains no valence-0 or valence-1 1-handles.

If the same hypothesis as above holds, except that the valence of each 2-handle of $\Delta$ is at least two, then the same conclusion holds except that in each case the upper bounds for $\rho_{1}\left(\Delta^{\prime}\right)$ are raised by one.

Proof. The assertion follows by induction on $C(\Delta)$. If $\eta$ is a valence-0 1-handle, then the manifold $M_{1}$ obtained by deleting $\eta$ is connected since $T$ is connected. By Lemma $1.10 M_{1}$ is non-elementary. If $\Delta_{1}$ is the induced handle structure on $M_{1}$, then $C\left(\Delta_{1}\right)=C(\Delta)-(0,1,0)$. Now let $M_{2}$ and $\Delta_{2}$ be obtained by deleting a 2-handle $\lambda$ of $\Delta_{1}$ whose lateral boundary intersects the 2 -sphere component of $\partial M_{1}$. Note that $\partial M_{2}$ is a union of tori and $M_{2}$ is non-elementary by Lemma 1.10. If each 1-handle of $\Delta_{2}$ is of valence at least 2 , then $\rho_{1}\left(\Delta_{2}\right)=\rho_{1}\left(\Delta_{1}\right)-$ valence $(\lambda) \leq \rho_{1}\left(\Delta_{1}\right)-3$. Otherwise either $\rho_{1}\left(\Delta_{2}\right)<\rho_{1}(\Delta)$ and there exists a valence $\leq 1$ 1-handle of $\Delta_{2}$ and the result follows by induction or $C\left(\Delta_{2}\right)<C(\Delta), \rho_{1}\left(\Delta_{2}\right)=\rho_{1}(\Delta)$ and $\Delta_{2}$ has either a valence-0 1-handle or at least two valence-1 1-handles. Again the result follows by induction.

If $\eta$ is a valence-1 1-handle, then canceling $\eta$ with its corresponding 2-handle creates a handle structure $\Delta_{1}$ on $M$ such that $C\left(\Delta_{1}\right)<C(\Delta)$. If $\rho_{1}\left(\Delta_{1}\right)=\rho_{1}(\Delta)$, then $\Delta_{1}$ either has a valence-0 1-handle or two valence-1 1-handles. Similarly, if $\rho_{1}\left(\Delta_{1}\right)=\rho_{1}(\Delta)-1$, then $\Delta_{1}$ has at least one valence $\leq 1$ 1-handle. In either case the result follows by induction.

The case that the valence of each 2 -handle of $\Delta$ is at least two follows similarly.

Lemma 3.10. Let $M$ be non-elementarily embedded in the compact hyperbolic 3manifold $N$, where $\partial M$ is a union of tori. Suppose that $M$ has a full handle structure $\Delta$ without 0-handles based on a component $T$ of $\partial M$ such that every 2-handle is of valence $\geq 3$. If either of the following are true then there exists a non-elementary $M^{\prime}$ with handle structure $\left(M^{\prime}, T^{\prime}, \Delta^{\prime}\right)$ such that $\rho_{1}\left(\Delta^{\prime}\right) \leq \rho_{1}(\Delta)-2$ and $\partial M^{\prime}$ is a union of tori:

(i) There exists a valence-1 2-handle $\sigma \subset N-\stackrel{\circ}{M}$ that can be added to $\Delta$.

(ii) There exists a disc $D \subset \partial M$ such that $\partial D$ is the union of two arcs $\alpha \cup \beta$, where $\beta$ lies in a lake and $\alpha$ is an essential arc in a 2-handle $\lambda$, i.e., $\alpha$ separates components of $\delta(\lambda) \cap(1-$ handles $)$ inside of $\delta(\lambda)$.

Proof. We first prove (i). By subdividing higher valence 2-handles we can assume that each 2-handle is of valence 3. By Lemma 3.9 we can assume that every 1handle of $\Delta$ is of valence $\geq 2$. Let $\Delta_{1}$ be the handle structure on the manifold $M_{1}$ 
obtained by attaching $\sigma$ to $\Delta$ along $\partial M$. Let $\eta$ denote the 1-handle which $\sigma$ meets. Let $\Delta_{2}$ and $M_{2}$ be obtained by deleting a 2-handle $\lambda \neq \sigma$ whose lateral boundary intersects the 2-sphere boundary component of $M_{1}$. By Lemma $1.10 M_{2}$ is nonelementary. Let $\Delta_{3}$ be obtained by cancelling $\sigma$ and $\eta$. If $\eta$ has valence $\geq 4$, then $\rho_{1}\left(\Delta_{3}\right) \leq \rho_{1}(\Delta)-2$. If so, then as in Lemma 3.6, reduce to a non-elementary $M_{4}$ with handle structure $\Delta_{4}$ on $\left(M_{4}, T\right)$ whose 1 and 2-handles are of valence $\geq 2$ and $\rho_{1}\left(\Delta_{4}\right) \leq \rho_{1}\left(\Delta_{3}\right)$.

Now assume that valence $\eta)=3$. If $\lambda$ runs over $\eta$ thrice, then $\eta$ is the unique 1-handle of $\Delta$ since $\Delta$ is full. This implies that $M_{2}=T \times I$ which is elementary, a contradiction.

Now assume that some 2-handle runs over $\eta$ twice. We can choose $\lambda$ to be this 2-handle. Let $\kappa$ denote the other 2-handle of $\Delta$ that attaches to $\eta$. Construct $M_{2}$ and $\Delta_{3}$ as above. Since valence $(\eta)=3$, we have $\rho_{1}\left(\Delta_{3}\right) \leq \rho_{1}(\Delta)-1$. If equality holds, then $\Delta_{3}$ has one valence-1 1-handle, one valence-2 2-handle (descended from $\kappa$ ) and all the other 2-handles have valence three. Indeed, the valence-1 1-handle $\eta^{\prime}$ of $\Delta_{3}$, when viewed in $\Delta$, is of valence 2 and is the other 1-handle to which $\lambda$ is attached. Now apply Lemma 3.9 to $\Delta_{3}$.

If no 2-handle runs over $\eta$ twice, then each 2-handle of $\Delta_{3}$ has valence at least two, independent of the choice of $\lambda$. Again $\rho_{1}\left(\Delta_{3}\right) \leq \rho_{1}(\Delta)-1$ and here equality implies that $\Delta_{3}$ has either one valence-0 1-handle or two valence-1 1-handles, so we can apply Lemma 3.9.

If valence $(\eta)=2$ and some 2-handle runs over it twice, then we can assume that this handle $\kappa$ is not $\lambda$. Indeed we can assume that $\lambda$ is chosen to run over a 1 -handle $\eta_{2}$ which is the other 2-handle run over by $\kappa$. Note that $\kappa$ becomes a valence-1 2-handle in $\Delta_{3}$. Let $\Delta_{4}$ be obtained by canceling this new $\kappa$ with $\eta_{2}$. Again $\rho_{1}\left(\Delta_{4}\right) \leq \rho_{1}(\Delta)-2$ if valence $\left(\eta_{2}\right) \geq 4$.

If valence $\left(\eta_{2}\right)=3$ and $\lambda$ runs over it twice, then $\rho_{1}\left(\Delta_{4}\right) \leq \rho_{1}(\Delta)-1$ and if equality holds, then $\Delta_{4}$ has a valence-1 1-handle and all its 2-handles are valence three. Thus, Lemma 3.9 applies.

If $\lambda$ runs over $\eta_{2}$ once, then $\rho_{1}\left(\Delta_{4}\right) \leq \rho_{1}(\Delta)-1$ and if equality holds, then either there is a valence-0 1-handle or two valence-1 1-handles. Further, all the 2-handles of $\Delta_{4}$ are at least valence- 2 and hence Lemma 3.9 applies.

If valence $\left(\eta_{2}\right)=2$, then $\rho_{1}\left(\Delta_{4}\right) \leq \rho_{1}(\Delta)$ and if equality holds, then either there is a valence-0 1-handle or two valence-1 1-handles. In this case all the 2-handles of $\Delta_{4}$ are of valence three and again Lemma 3.9 applies.

We now prove (ii). By pushing $D$ out of $M$ we obtain a properly embedded disc $E$ in $\overline{N \backslash M}$ with $\partial E=\partial D$. If $L$ is the component of $\delta(\lambda)$ intersected by $D$ and $D \cap L$ can be isotoped rel $\partial L$ into a 1-handle of $\Delta$, then $D$ becomes a valence-1 2-handle that can be added to $\Delta$. Now apply (i). Otherwise, we can introduce a new valence-2 1-handle $\eta$ to subdivide $\lambda$ into two valence $\geq 32$-handles which meet along $\eta$ such that $\partial D$ runs over $\eta$ once. This does not change $\rho_{1}$. Again, (i) applies. 
Lemma 3.11. Let $M$ be a non-elementarily embedded compact 3-manifold in the compact hyperbolic 3-manifold $N$ with $\partial M$ a union of tori, and let $\Delta$ be a handle structure for $M$ based on $R \subset \partial M$. If there exists a valence $\geq 31$-handle $\eta$ of $\Delta$ which attaches to a 0 -handle $\zeta$, then there exists a non-elementary embedding $M^{\prime} \rightarrow N$ and a handle structure $\Delta^{\prime}$ based on $R^{\prime} \subset \partial M^{\prime}$ such that $\rho_{1}\left(\Delta^{\prime}\right)<\rho_{1}(\Delta)$. Here either $\left(M^{\prime}, R^{\prime}\right)=\underset{\circ}{(M, R)}$ or $M^{\prime}=M-\stackrel{\circ}{V}$ and $R^{\prime}=R \cup \partial V$ where $V$ is an embedded solid torus in $M$.

Proof. If $\eta$ also attaches to either the base or a 0 -handle distinct from $\zeta$, then cancelling $\eta$ with $\zeta$ gives rise to a handle structure $\Delta^{\prime}$ on $(M, R)$ with $\rho_{1}\left(\Delta^{\prime}\right)<\rho_{1}(\Delta)$. If $\eta$ attaches only to $\zeta$, then let $M^{\prime}$ be obtained by hollowing out $\eta$ and $\zeta$ and $V$ the hollowed out solid torus.

Lemma 3.12. Let $N$ be a compact hyperbolic 3-manifold. If $(M, T, \Delta)$ is a full internal weak Mom- $n$ structure on $N$ and $M$ is reducible then there exists $\left(M_{p}, T_{p}, \Delta_{p}\right)$, a weak internal Mom- $k$ structure on $N$ such that $k=\rho_{1}\left(\Delta_{p}\right) \leq \rho_{1}(\Delta)=n-2$.

Proof. Proof by induction on $\rho_{1}(\Delta)$. Recall that $T$ is connected, since Mom-n and weak Mom-n structures are based on a single torus. We first consider the case that $T$ is incompressible in $N$. Let $F$ be a least-weight normal reducing 2-sphere. Incompressibility implies that each component of $F \cap T \times\{1\}$ is inessential in $T \times\{1\}$ and least-weight implies that each of these circles bounds a disc in $F \cap(T \times I)$. Let $M_{0}$ and $M_{0}^{\prime}$ be the components of $M$ split along $F$. By Lemma 1.10 and the irreducibility of $N$, exactly one of $M_{0}, M_{0}^{\prime}$ is non-elementary. We let $M_{0}$ (resp. $M_{0}^{\prime}$ ) denote the non-elementary (resp. elementary) component with $\Delta_{0}$ (resp. $\Delta_{0}^{\prime}$ ) its induced structure. Note that $T \subset M_{0}$.

Let $X$ denote the union of the islands and bridges of $\Delta, Y=X \cap M_{0}$ and $Y^{\prime}=X \cap M_{0}^{\prime}$.

We now show that $\rho_{1}\left(\Delta_{0}^{\prime}\right)>0$ and hence $\rho_{1}\left(\Delta_{0}\right) \leq \rho_{1}(\Delta)-1$, since $\rho_{1}\left(\Delta_{0}\right)+$ $\rho_{1}\left(\Delta_{0}^{\prime}\right)=\rho_{1}(\Delta)=n$. Assuming the contrary we show that $M_{0}^{\prime}$ is an $I$-bundle. Indeed, since $F$ is normal, each component of intersection of $M_{0}^{\prime}$ with a 2-handle is a product. Since $\rho_{1}\left(\Delta_{0}^{\prime}\right)=0$, the same is true for the 1-handles and these local product structures match up where appropriate. Thus each component of $Y^{\prime}$ is an annulus. These annuli extend to $D^{2} \times I^{\prime}$ s in $T \times[0,1]$. Here a component $E$ of $D^{2} \times\{0,1\}$ is a lake if $\partial E$ is the boundary of a lake (this uses fullness) and $E \subset F$ otherwise. Observe that $M_{0}^{\prime}$ a nontrivial I-bundle since $F$ is an essential 2-sphere. However, if $M_{0}^{\prime}$ is not a trivial I-bundle, then $F$ does not bound a 3-ball to the $M_{0}^{\prime}$ side, which implies that $M_{0}$ lies in a 3-ball, a contradiction.

A similar argument shows that some component of $Y$ is not an annulus and further some 2-handle $\sigma$ of $\Delta_{0}$ faces $F$ and attaches to a valence $\geq 31$-handle of $\Delta_{0}$. Delete $\sigma$ from $\Delta_{0}$ to obtain $\left(M_{1}, T, \Delta_{1}\right)$ with $\rho_{1}\left(\Delta_{1}\right) \leq \rho_{1}\left(\Delta_{0}\right)-1$. Now apply Lemma 3.6. 
We next consider the case that $T$ is compressible in $M$. Let $F$ be a least-weight compressing disc for $T$. Note that $F \cap T \times I$ consists of discs, and a single annulus that is isotopic to a vertical one in $T \times I$. If $M_{1}$ is $M$ split along $F$ with induced handle structure $\Delta_{1}$ and $A$ is $T$ split along $F \cap T$, then $\Delta_{1}$ is based on $A$. By Lemma 1.10 $M_{1}$ is non-elementary. Note that $\partial M_{1}$ contains a 2 -sphere $S$ that bounds a 3-ball to the outside of $M_{1}$ and so $M$ is obtained by attaching a possibly knotted 1-handle to $M_{1}$ inside of that ball. This implies that $T$ lies in a 3-cell. If that 1-handle is parametrized by $D^{2} \times[1,10]$, then we can assume that $M_{1}$ is obtained by deleting $D^{2} \times(4,5)$.

Let $M_{2}$ be obtained by attaching 2-handles $\sigma_{1}, \sigma_{2}$ to $\partial M_{1}$ along the components of $\partial A \times I$, i.e., along thin neighborhoods of the curves $\partial D^{2} \times\{4,5\}$. Note that $\partial M_{2}$ has three 2-sphere components, two of which are inside of the just added caps. Attach a 3-cell $B$ to the third 2-sphere to obtain the manifold $M_{3}$. The induced handle structure $\Delta_{3}$ on $M_{3}$ is classical. Here $A \times I \cup \sigma_{1} \cup \sigma_{2} \cup B$ comprise one of the 0-handles of $\Delta_{3}$.

Since $\Delta$ is full $F \cap X$ must cross a 1-handle of valence at least three, where $X$ is defined as previously. Thus the lateral surface of some 2-handle of $\Delta_{3}$ faces a 2-sphere of $\partial M_{3}$ and attaches to a 1-handle of valence $\geq 3$. Delete this 2-handle to obtain the non-elementary $M_{4}$ with handle structure $\Delta_{4}$ which satisfies $\rho_{1}\left(\Delta_{4}\right)+1 \leq \rho_{1}(\Delta)$. Delete another 2-handle to create $M_{5}$ and $\Delta_{5}$ such that $\partial M_{5}$ is a union of tori. Next apply Lemma 3.7 to produce $\left(M_{6}, \Delta_{6}\right)$. Let $\eta$ be a valence $\geq 31$-handle of $\Delta_{6}$, which exists by 3.5. Next hollow out the 0 -handle and $\eta$ to obtain $\left(M_{7}, T_{7}, \Delta_{7}\right)$ with $\rho_{1}\left(\Delta_{7}\right) \leq \rho_{1}\left(\Delta_{5}\right)-1$. Finally apply Lemma 3.6 to obtain the desired weak Mom- $k$ structure.

We consider the last case, that $T$ is incompressible in $M$ but not in $N$. Let $F$ be a least-weight essential normal 2-sphere for $\Delta$. Incompressibility implies that $F \cap(T \times I)$ is a union of discs. If $T$ does not lie in the ball $B$ bounded by $F \subset N$, then proceed as in the first case of the proof. Otherwise let $M_{0}$ and $M_{0}^{\prime}$ be the components of $M$ split along $F$, with $M_{0}^{\prime}$ the component lying in $B$. Let $\Delta_{0}, \Delta_{0}^{\prime}$ denote the corresponding handle structures on $M_{0}$ and $M_{0}^{\prime}$. Since $\Delta$ is full and some component of $T \times 1 \cap M_{0}^{\prime}$ is nonplanar, it follows that $\rho_{1}\left(\Delta_{0}^{\prime}\right) \geq 1$ and hence $\rho_{1}\left(\Delta_{0}\right) \leq \rho_{1}(\Delta)-1$. Now complete the proof as in the previous paragraph, by first obtaining a classical handle structure $\left(M_{1}, \Delta_{1}\right)$ by deleting a 2-handle from $\Delta_{0}$, then applying Lemma 3.7, then reducing $\rho_{1}$ by hollowing out a 0 -handle and a valence $\geq 3$ 1-handle and finish by applying Lemma 3.6.

Definition 3.13. If $\Delta$ is a handle structure on $M$, then the sheets of $\Delta$ are the connected components of the space $S$ which is the union of the 2-handles and the valence-2 1-handles. So sheets are thickened surfaces which are attached to a 3-manifold along their thickened boundaries. The valence of a sheet is the number of times the boundary runs over 1-handles counted with multiplicity. 
Lemma 3.14. Let $N$ be a compact hyperbolic 3-manifold and $f: M \rightarrow N$ a nonelementary embedding where $\partial M$ is a union of tori. Let $\Delta$ be a full handle structure on $M$ with no 0-handles based on a component $T$ of $\partial M$ such that the valence of each 2-handle is at least 3. If some sheet $S$ of $\Delta$ is not a thickened disc then there exists a handle structure $\left(M^{\prime}, T^{\prime}, \Delta^{\prime}\right)$ such that $M^{\prime}$ is non-elementary, $\partial M^{\prime}$ is a union of tori and $\rho_{1}\left(\Delta^{\prime}\right) \leq \rho_{1}(\Delta)-1$. If for every such $\left(M^{\prime}, T^{\prime}, \Delta^{\prime}\right)$ equality must hold, then $S$ is a thickened Möbius band and there exists such a $\left(M^{\prime}, T^{\prime}, \Delta^{\prime}\right)$ such that $\Delta^{\prime}$ is full and $T^{\prime}=T$ (in particular $T^{\prime}$ is connected $)$.

Proof. By Lemmas 3.9 and 3.12 we can assume that each 1-handle of $\Delta$ has valence $\geq 2$ and $M$ is irreducible. If $M_{1}$ denotes the manifold obtained by deleting the sheet $S$, then $\chi(M)=\chi\left(M_{1}\right)+\chi(S)$. Since $\chi(M)=0$, if $\chi(S)<0$, then $\partial M_{1}$ contains a 2-sphere and hence $M$ is either elementary or reducible, a contradiction.

Now assume that $\chi(S)=0$; note that $M_{1}$ is non-elementary by Lemma 1.10 . In this case $S$ is either an annulus $\times I$ or a non-trivial $I$-bundle over a Möbius band. If $S$ contains either at least two 2-handles or a single 2-handle of valence at least 4 , then $\operatorname{valence}(S) \geq 2$. If valence $(S)>1$, then either $\rho_{1}\left(\Delta_{1}\right) \leq \rho_{1}(\Delta)-2$, where $\Delta_{1}$ is the induced structure on $M_{1}$ or $\rho_{1}\left(\Delta_{1}\right)=\rho_{1}(\Delta)-1$ and Lemma 3.9 applies. If $S$ is a valence- 1 annulus $\times I$, then $\Delta$ is not full. If $S$ is a thickened Möbius band of valence 1 , then $\rho_{1}\left(\Delta_{1}\right)=\rho_{1}(\Delta)-1$. Note that $\Delta_{1}$ is based on $T$.

We consider the case that $\Delta_{1}$ is not full and valence $(S)=1$. Here $S$ runs over the 1-handle $\eta$ of $\Delta_{1}$ and is attached to the component $R$ of $\partial M_{1}$. After isotopy the core $\alpha_{1}$ of the attaching annulus of $S$ crosses the core of the annular lake $L_{1}$ of $\Delta_{1}$ exactly once. Let $\Delta_{2}$ be the handle structure on $M_{1}$ obtained by turning $\Delta_{1}$ upside down. By Lemma $2.11 \rho_{1}\left(\Delta_{2}\right)=\rho_{1}\left(\Delta_{1}\right)$. In the switch $\eta$ becomes a 2-handle $\sigma$. The lake $L_{1}$ corresponds to a lake $L_{2} \subset R \times\{1\}$ of $\Delta_{2}$ and $\alpha_{1}$ corresponds to a curve $\alpha_{2} \subset R \times\{1\}$ which crosses both the attaching curve of $\sigma$ exactly once and the core of $L_{2}$ once. Let $\left(M_{3}, \Delta_{3}\right)$ be obtained from $\left(M_{1}, \Delta_{2}\right)$ by splitting $M_{1}$ along an annulus connecting the core of $L_{2}$ to $T$. Then $\Delta_{3}$ is based on an annulus $C$ together with a possibly empty union $U=\partial M_{1} \backslash(T \cup R)$ of tori . Here $C=R \backslash \stackrel{\circ}{L_{2}}$. Since $\sigma$ crosses $\alpha_{2}$, an essential arc in $C \times\{1\}$, once and the valence of each 1-handle of $\Delta_{3}$ is at least 3 (since that was true of 2-handles of $\Delta_{2}$ ), we can apply Lemma 3.8 to conclude that there exists a handle structure $\left(M_{3}, U, \Delta_{4}\right)$ with $\rho_{1}\left(\Delta_{4}\right)<\rho_{1}\left(\Delta_{3}\right)$. If $U=\emptyset$, then $\Delta_{4}$ is a classical handle structure. In that case, hollowing out produces $\left(M_{5}, T_{5}, \Delta_{5}\right)$ with $M_{5}$ non-elementary, $\partial M_{5}$ a union of tori, $T_{5}$ a torus component of $\partial M_{5}$ and $\rho_{1}\left(\Delta_{5}\right) \leq \rho_{1}\left(\Delta_{4}\right)$.

The next three lemmas deal with the case that $M$ contains an essential annulus.

Lemma 3.15. Let $(M, T, \Delta)$ be an internal Mom-n structure on the compact hyperbolic manifold $N$. Assume that every sheet of $\Delta$ is a thickened disc and $\Delta$ is full. 
If there exists a properly embedded essential annulus $A \subset M$ with boundary components respectively on $T$ and a component of $\partial M \backslash T$, then there exists a handle structure $\left(M^{\prime}, T^{\prime}, \Delta^{\prime}\right)$ such that $M^{\prime}$ is non-elementary, $\partial M^{\prime}$ is a union of tori and $\rho_{1}\left(\Delta^{\prime}\right) \leq \rho_{1}(\Delta)-2$.

Proof. By Lemma 3.12 we can assume that $M$ is irreducible and hence by Lemma 1.11 $\partial M$ is incompressible. Since all sheets are discs we can cancel valence-2 1-handles with 2-handles to obtain a full handle structure $\left(M, T, \Delta_{1}\right)$ without 0 -handles such that $\rho_{1}\left(\Delta_{1}\right)=\rho_{1}(\Delta)$ and every 1 and 2-handle is of valence $\geq 3$. Since $A$ is $\pi_{1}$ injective and $\partial M$ is incompressible, $A$ is also boundary incompressible. Therefore by Lemma $2.3 A$ is isotopic to a normal surface. By replacing $A$ if necessary, we can assume that $A$ is least-weight among all properly embedded normal essential annuli connecting $T$ to another boundary component $J$ of $M$. Since $A$ is least weight, $A \cap T \times I$ is a union of discs and a single annulus.

Let $A_{0}$ (resp. $A_{1}$ ) denote $A \cap T$ (resp. $A \cap J$ ). Let $M_{2}$ denote the manifold obtained by splitting $M$ along $A$ and let $\Delta_{2}$ denote the handle structure induced from $\Delta_{1}$. As in $\S 2$, those components of $T \times I$ split along $A$ which are 3-balls are the 0-handles of $\Delta_{2}$. The remaining component is homeomorphic to $B^{\prime} \times I$, where $\left(B^{\prime} \times I\right) \cap T=B^{\prime} \times 0$ is $T$ split along $A_{0}$ and hence $\Delta_{2}$ is based on $B^{\prime} \subset T$. Note that $M_{2}$ is connected. By Lemma $1.10, M_{2}$ is non-elementarily embedded in $N$.

As in [Mv1], if $\eta$ is a 1-handle of $\Delta_{1}$ and if $\left\{\eta_{i}\right\}$ denotes the 1-handles of $\Delta_{2}$ which descend from $\eta$, then $\sum_{i} \max \left(\left(\operatorname{valence}\left(\eta_{i}\right)-2\right), 0\right) \leq$ valence $(\eta)-2$ with equality if and only if $A_{1}$ does not run over $\eta$.

Claim. If counting with multiplicity $A_{1}$ runs over more than one 1-handle, then the conclusion of the lemma holds.

Proof of Claim. This is immediate if $A_{1}$ runs over distinct 1-handles. Now assume that $A_{1}$ runs over a unique 1-handle $\eta$. Let $R_{1}, \ldots, R_{n}$ be the components of $A \cap \eta$ which nontrivially intersect $A_{1}$. Let $C_{i}$ and $D_{i}$ denote the closure of the components of $\delta \eta \backslash R_{i}$.

If some $R_{i}$ intersects $\partial M$ in two components and say $C_{i}$ intersects the attaching zone of exactly one 2-handle $\sigma$ and $\sigma \cap C_{i}$ is connected, then viewed in $\Delta_{2}, \sigma$ attaches to a valence-1 1-handle $\eta_{1}$ descended from $\eta$. Furthermore, $A \cap \sigma=\emptyset$ otherwise condition (ii) of Definition 2.1 is violated. Since the valence of every 1-handle of $\Delta_{1}$ is $\geq 3$ it follows that in $\Delta_{2}, \sigma$ only attaches to valence $\geq 31$-handles except for those 1-handles descended from $\eta$. Therefore, after cancelling $\sigma$ with $\eta_{1}$, either $\rho_{1}$ is reduced or viewed in $\Delta_{1}, \sigma$ only attaches to $\eta$ and both $\eta$ and $\sigma$ have valence 3 . In that case, since $\Delta_{1}$ is full, $\eta$ is the unique 1-handle of $\Delta$ and $\rho_{1}\left(\Delta_{2}\right)=0$. This contradicts Lemma 3.5.

If some $R_{i}$ intersects $\partial M$ in two components and each of $C_{i}$ and $D_{i}$ intersect attaching annuli of 1-handles of $\Delta_{1}$ in at least two components, then $\rho_{1}\left(\Delta_{2}\right) \leq$ $\rho_{1}\left(\Delta_{1}\right)-2$. 
We now assume that each $R_{i}$ intersects $\partial M$ in exactly one component. If two such $R_{i}$ 's are not normally isotopic in $\eta$, then again $\rho_{1}\left(\Delta_{2}\right) \leq \rho_{1}\left(\Delta_{1}\right)-2$. Therefore we can assume that all the $R_{i}$ 's are normally parallel. Since all lakes are discs, it follows that if $L$ is the lake with $L \cap A \neq \emptyset$, then $L \cap A$ equals $n$ parallel arcs. Since $A_{1}$ is connected, $n=1$.

We now assume that $A_{1}$ runs over a unique 1-handle $\eta$ and it does so with multiplicity one. This implies that $\rho_{1}\left(\Delta_{2}\right)=\rho_{1}\left(\Delta_{1}\right)-1$. Also $A_{1}$ is the union of two arcs $\alpha$ and $\beta$, where $\alpha$ lies in a 1-handle of $\Delta_{1}$ and $\beta$ lies in a lake of $\Delta_{1}$.

Our $A$ has an induced handle structure $\Phi$ based on $A_{0}$ as follows. The base $Z$ consists of the annular component of $A \cap T \times I$, the 0 -handles consist of the disc components of $A \cap T \times I$, the 1-handles (resp. 2-handles) consist of the intersections of $A$ with the 1-handles (resp. 2-handles). Since $\Delta_{1}$ has no annular lakes and $A$ is an annulus, $\Phi$ contains at least one 0 -handle. We abuse notation by viewing 0 -handles of $\Phi$ also as properly embedded discs in $T \times I$.

Give $A$ a transverse orientation. Call a 0 -handle $v$ of $\Phi$ plus (resp. minus) if the transverse orientation of the disc $v \subset T \times I$ points away from (resp. towards) $T$. Each disc $v$ separates in $T \times I$ a 3-ball $v_{B}$ from $T$. Let $v_{D}$ denote $v_{B} \cap T \times\{1\}$.

Viewed in $A, \beta$ lies in the boundary of either the base of $\Phi$ or a 0 -handle $v$ of $\Phi$. We consider the case that $\beta$ lies in a 0 -handle $v$. Since $A$ is least weight, the disc $v_{D}$ contains a bridge $b$ in its interior and this bridge is not parallel to $\beta$. In fact by (ii) of Definition 2.1, if $P$ and $Q$ are the islands in $T \times\{1\}$ corresponding to $\eta$, there exists bridges $b_{1}, b_{2}$ emanating from each of $P$ and $Q$ lying interior to $v_{D}$. Since $A_{1}$ runs over a unique 1-handle it follows that $b_{1} \neq b_{2}$, else $\Delta_{1}$ has a valence-1 2-handle. Now suppose that $b_{1} \neq b_{2}$. If $v_{D} \cap w_{D}=\emptyset$ for all 0 -handles $w \neq v$ of $\Phi$, then Lemma 3.11 applies to the 0 -handle $v_{B}$ of $\Delta_{2}$ and the Lemma is proved. Indeed, $v_{B}$ can cancel with a valence $\geq 31$-handle $\kappa_{1}$ of $\Delta_{2}$ which descends from a 1-handle $\kappa \neq \eta$ of $\Delta_{1}$, one of whose islands intersects $b_{1}$. Otherwise, $v_{B}$ is split into balls by the various 0-handles of $\Phi$ and Lemma 3.11 applies to one of these balls.

From now on we assume that $\beta$ lies in the base $Z$ of $\Phi$. Let $X \subset T \times 1$ denote the union of the islands and bridges of $\Delta_{1}$. An argument similar to one in the previous paragraph shows that for each 0-handle $v$ of $\Phi$ either $\partial v$ is boundary parallel in $X$ or Lemma 3.11 applies.

We now assume that if $v$ is a 0 -handle of $\Phi$, then $\partial v$ is boundary parallel in $X$. This implies

i) if $v \neq w$ are 0 -handles of $\Phi$, and $v_{B} \subset w_{B}$, then $\partial v$ and $\partial w$ are normally parallel in $X$,

ii) no 1-handle of $\Phi$ connects a plus 0-handle to a minus 0-handle of $\Phi$ and

iii) if $v^{0}$ and $v^{1}$ are two 0 -handles of $\Phi$ that are connected by a 1-handle, then $v_{B}^{0} \cap v_{B}^{1}=\emptyset$.

It follows from (i)-(iii) and the compactness of $F$ that 
iv) there do not exist 0 -handles $w^{0}, w^{1}, \ldots, w^{n}$ of $\Phi$ such that for $i=1,2, \ldots$, $n-1, w^{i}$ is connected to $w^{i+1}$ by a 1 -handle and $w_{B}^{n} \subset w_{B}^{0}$.

Let $Z_{0}$ and $Z_{1}$ denote the components of $\partial Z$ where $Z_{0}=A_{0}$. Since $A_{1}$ runs over a unique 1-handle, $Z_{1} \cap \partial M=\beta$. Therefore $Z_{1}=\beta \cup \beta_{1}$ where $\beta_{1}$ is a properly embedded arc in $A$. Let $H \subset A$ be the disc $\overline{A \backslash Z}$. Define an equivalence relation on 0-handles of $\Phi$ generated by the relation that $v \sim w$ if $v$ and $w$ are connected by a 1-handle of $\Phi$. Given a class [v], let $E_{v}^{\prime} \subset H$ be the union of all 0-handles of $\Phi$ lying in $[v]$ together with all 1-handles of $\Phi$ which attach to discs in $[v]$. Let $E_{v} \subset H$ be the largest disc bounded by an arc in $\beta_{1}$ and an $\operatorname{arc} \gamma$ in $\partial E_{v}^{\prime}$. Notice that if $E_{v} \cap E_{w} \neq \emptyset$, then either $E_{v} \subset E_{w}$ or $E_{w} \subset E_{v}$ with both holding if and only if $[v]=[w]$.

Let $[v]$ be a class with $E_{v}$ innermost. A small neighborhood $E \subset H$ of $E_{v}$ is a disc whose boundary is the union of two $\operatorname{arcs} \phi$ and $\psi$ where $\phi$ is a proper arc in a 2-handle of $\Phi$ and $\psi$ lies in $Z_{1}$. Furthermore $E$ non trivially intersects exactly one $E_{v}^{\prime}$. By (ii)-(iv) above, if $v, w$ are 0 -handles of $\Phi$ lying in $E$, then they are of the same parity and $v_{B} \cap w_{B}=\emptyset$.

Since every 0-handle of $\Phi$ is normally boundary parallel and iv) holds, $E$ can be normally isotoped with respect to $\Delta_{1}$ to a disc $G \subset \partial M$ such that $\partial G$ is a union of two arcs, one lying in a lake and the other in a 2-handle. Now apply (ii) of Lemma 3.10 to $\Delta$.

Lemma 3.16. Let $(M, T, \Delta)$ be a full internal Mom-n structure on the compact hyperbolic 3-manifold $N$. Suppose that every sheet of $\Delta$ is a disc and there exists an essential embedded annulus $A$ with $\partial A \cap T=\emptyset$. Then there exists a non-elementary embedding $M^{\prime} \rightarrow N$ with handle structure $\left(M^{\prime}, T^{\prime}, \Delta^{\prime}\right)$ where $\partial M^{\prime}$ is a union of tori and $\rho_{1}\left(\Delta^{\prime}\right) \leq \rho_{1}(\Delta)-2$.

Proof. Assume that $M$ is irreducible and therefore $\partial M$ is incompressible. Again by canceling 1 and 2-handles we can pass to a handle structure $\Delta_{1}$ of $(M, T)$ which is full, has the same $\rho_{1}$ and every 1 and 2-handle has valence $\geq 3$. Let $A$ be a least-weight normal essential annulus with $A \cap T=\emptyset$. By Lemma 3.15 we can assume that no essential annulus connects $T$ to a component of $\partial M \backslash T$ and hence $A \cap T \times I$ is a union of discs. Let $M_{2}$ be the non-elementary component of $\mathrm{M}$ split along $A$ and let $\left(M_{2}, T_{2}, \Delta_{2}\right)$ be the induced handle structure. As in the previous proof, $\rho_{1}\left(\Delta_{2}\right) \leq \rho_{1}\left(\Delta_{1}\right)-1$. Furthermore, the argument of the Claim implies that either the lemma is true or counted with multiplicity $\partial A$ traverses a single 1-handle $\eta$ in two normally parallel components; furthermore, $A \cap \eta$ consists of two normally parallel discs which contain $\eta \cap \partial A$. There are two components since $|\partial A|=2$. If $\rho_{1}\left(\Delta_{2}\right)=\rho_{1}\left(\Delta_{1}\right)-1$, then as in the proof of Lemma 3.12 there is a non-elementary component of $M \backslash A$ and the closure of that component is a non-trivial $I$-bundle. Since $A$ is an annulus, it must be a non-trivial $I$-bundle over a Möbius band $\hat{A}$. 
We now assume that $A$ normally double covers a Möbius band $\hat{A}$ and $\partial \hat{A}$ runs over a single 1-handle $\eta$ of $\Delta$ with multiplicity one. In that case $\partial \hat{A}$ is the union of two $\operatorname{arcs} \alpha$ and $\beta$ where $\alpha \subset \eta$ and $\beta$ is properly embedded in a lake of $\Delta$. Note that $T \subset \partial M_{2}$.

Let $\Phi$ be the induced handle structure on $A$. In this case, $\Phi$ is a classical handle structure. Therefore $\beta \subset \partial v$, where $v$ is a 0 -handle of $\Delta_{1}$. As in the proof of Lemma 3.15, there exists a valence $\geq 31$-handle of $\Delta_{2}$ that attaches to a 0 -handle of $\Delta_{2}$ and hence by Lemma 3.11 there exists $\left(M_{3}, T_{3}, \Delta_{3}\right)$ such that $M_{3}$ is nonelementary, $\partial M_{3}$ is a union of tori and $\rho_{1}\left(\Delta_{3}\right)<\rho_{1}\left(\Delta_{2}\right)$.

Lemma 3.17. Let $(M, T, \Delta)$ be a full internal Mom-n structure on the compact hyperbolic 3-manifold $N$ such that every sheet is a disc. Suppose that there exists an essential embedded annulus $A$ with $\partial A \subset T$. Then there exists a handle structure $\left(M^{\prime}, T^{\prime}, \Delta^{\prime}\right)$ where $M^{\prime}$ is non-elementary, $\partial M^{\prime}$ is a union of tori and $\rho_{1}\left(\Delta^{\prime}\right) \leq$ $\rho_{1}(\Delta)-2$.

Proof. By Lemma 3.12, Lemma 3.15 and Lemma 3.16 we can assume that $M$ is irreducible, $\partial M$ is incompressible and all essential annuli must have both boundary components in $T$. Let $(M, R, \Sigma)$ be the dual handle structure, where $R=\partial M \backslash T$. Note that $\rho_{1}(\Sigma)=\rho_{1}(\Delta), \Sigma$ is full and each 1-handle of $\Sigma$ is of valence 3, though $\Sigma$ may have valence-2 2-handles.

Let $A$ be an essential annulus, least weight with respect to $\Sigma$. Since each essential annulus has its entire boundary in $T, A \cap R \times I$ is a union of discs. Let $M_{1}$ be $M$ split along $A$ with $\left(M_{1}, R, \Sigma_{1}\right)$ the induced structure. As before $\rho_{1}\left(\Sigma_{1}\right)<\rho_{1}(\Sigma)$. Let $M_{2}$ be the non-elementary component of $M_{1}$ and $\left(M_{2}, R_{2}, \Sigma_{2}\right)$ the induced handle structure.

Claim. Either the conclusion of the lemma holds or $\partial A$ runs over a single one handle $\eta$ of $\Sigma$ and $A \cap \eta$ contains two normally parallel discs which contain $\partial A \cap \eta$. Furthermore each such disc intersects $\partial M$ in a single arc.

Proof of Claim. In contrast to the previous claim both components of $\partial A$ are disjoint from the base as opposed to just one, $\Sigma$ may have valence-2 2-handles and $R$ may have more components than $T$. Note that the number of components of $T$ did not play a role in the proof. The proof is immediate if $\partial A$ runs over distinct 1-handles. Now assume that $\partial A$ runs over a unique 1-handle $\eta$. Let $R_{1}, \cdots, R_{n}$ be the components of $A \cap \eta$ which nontrivially intersect $\partial A$. Let $C_{i}$ and $D_{i}$ denote the closure of the components of $\delta \eta \backslash R_{i}$.

The proof follows exactly as in the previous claim except in the case that some $R_{i}$ intersects $\partial M$ in two components and say $C_{i}$ intersects the attaching zone of but one 2-handle $\sigma$ and $\sigma \cap C_{i}$ is connected. The lemma holds as before unless, as viewed in $\Sigma, \sigma$ only attaches to $\eta$, valence $(\eta)=3$, and valence $(\sigma)=2$. Let $\sigma^{\prime}$ be the other 2handle of $\Sigma$ that attaches to $\eta$. If $\sigma \subset M_{2}$, then the lemma follows from Lemma 1.10 
since $\sigma$ attaches to a valence-1 1-handle $\eta_{1}$ of $\Sigma_{2}$. Otherwise there exists a 2-handle $\sigma_{2}$ of $\Sigma_{2}$ descended from $\sigma^{\prime}$ that attaches to a valence-1 1-handle of $\Sigma_{2}$. Now apply Lemma 1.10 .

The proof now follows as in the previous lemma. I.e., either $A$ double covers a Möbius band $\hat{A}$ or $\rho_{1}\left(\Sigma_{2}\right) \leq \rho_{1}(\Sigma)-2$ and in the former case after canceling a 0 -handle of $\Sigma_{2}$ with a valence $\geq 31$-handle of $\Sigma_{2}, \rho_{1}$ is reduced.

Lemma 3.18. Let $(M, T, \Delta)$ be a full internal Mom-n structure on the compact hyperbolic 3-manifold $N$. If $M$ is not hyperbolic, then either there exists a full internal Mom-k structure $\left(M^{\prime}, T, \Delta^{\prime}\right)$ with $k<n$ and $M^{\prime}$ hyperbolic or there exists a weak Mom-r structure structure $\left(M^{\prime}, B^{\prime}, \Delta^{\prime}\right)$ with $r \leq n-2$.

Proof. Proof by induction on $\rho_{1}(\Delta)$. By Lemmas 3.12, 3.14, 3.15, 3.16, 3.17, and 3.6 it suffices to consider the case that $M$ is irreducible and annular and every sheet is a disc.

By Thurston [Th2], if $M$ is annular but not hyperbolic, then it contains an essential torus. Let $F$ be a least-weight essential torus. Since there are no essential annuli, $F \cap T \times I$ is a union of discs.

Let $M_{1}$ denote the component of $M$ split along $F$ which contains $T$ and let $M_{1}^{\prime}$ denote the other component. Let $\Delta_{1}$ and $\Delta_{1}^{\prime}$ denote the induced handle structures. Note that $\Delta_{1}$ is based on $T, \Delta_{1}^{\prime}$ is a classical structure and $\rho_{1}\left(\Delta_{1}\right)+\rho_{1}\left(\Delta_{1}^{\prime}\right)=\rho_{1}(\Delta)$. Let $X \subset T \times\{1\}$ be the union of the islands and bridges of $\Delta, Y=M_{1}^{\prime} \cap X$ and $Z=M_{1} \cap X$.

By Lemma 1.10 one of $M_{1}$ or $M_{1}^{\prime}$ is non-elementary. If $M_{1}$ is elementary, $\rho_{1}\left(\Delta_{1}\right) \geq 1$ since $Z$ is nonplanar. By Lemma 3.7 there exists a handle structure $\Delta_{2}$ on $M_{1}^{\prime}$ such that $\rho_{1}\left(\Delta_{2}\right) \leq \rho_{1}\left(\Delta_{1}^{\prime}\right), \Delta_{2}$ has a unique 0-handle, and each 1-handle is of valence $\geq 2$. Since $M_{1}^{\prime}$ is non-elementary, some 1-handle is of valence $\geq 3$. Now apply Lemma 3.11 .

From now on assume that $M_{1}$ is non-elementary. If $\rho_{1}\left(\Delta_{1}^{\prime}\right)=0$, then as in Lemma 3.12, $M_{1}^{\prime}$ is a non trivial I-bundle, hence a non trivial $I$-bundle over a Klein bottle, which is impossible in a hyperbolic 3-manifold. Hence $\rho_{1}\left(\Delta_{1}\right)<\rho_{1}(\Delta)$.

Since $\Delta$ is full and $F \cap T \times I$ are discs, it follows that $\Delta_{1}$ is full, though it may have 0 -handles. If some valence $\geq 31$-handle attaches to a 0 -handle of $\Delta_{1}$, then the lemma follows from Lemma 3.11. Otherwise, cancel the 0-handles with 1-handles to obtain a full handle structure $\left(M_{1}, T, \Delta_{2}\right)$. Cancel the valence-1 2-handles of $\Delta_{2}$ with their associated 1-handles and if necessary repeat the process, to obtain the handle structure $\left(M_{3}, T, \Delta_{3}\right)$. Note that either $\Delta_{3}$ is a full weak Mom-r structure or $\rho_{1}\left(\Delta_{3}\right)<\rho_{1}\left(\Delta_{1}\right)$. 


\section{From Mom-n to hyperbolic Mom-n}

The main result of this section is the following:

Theorem 4.1. If $(M, T, \Delta)$ is a full internal Mom-n structure on the compact hyperbolic 3-manifold $N$ and $n \leq 4$, then there exists a full internal Mom- $k$ structure $\left(M^{\prime}, T^{\prime}, \Delta^{\prime}\right)$ on $N$ where $M^{\prime}$ is hyperbolic and $k \leq n$.

For general $n$, either there exists a full internal Mom- $k$ structure $\left(M^{\prime}, T^{\prime}, \Delta^{\prime}\right)$ in $N$ with $k \leq n$ and $M$ hyperbolic or there exists a general based internal Mom- $k$ structure $\left(M^{\prime}, B^{\prime}, \Delta^{\prime}\right)$ on $N$ such that $k \leq n-2, M^{\prime}$ is hyperbolic and each component of $B^{\prime}$ is either a component of $\partial M^{\prime}$ or an essential annulus in a component of $\partial M$.

Remark 4.2. Recall that in this paper all general based handle structures $(M, B, \Delta)$ have $B$ being a union of components of $\partial M$ and essential annuli of $\partial M$ except for brief moments when disc components are created. But these are immediately transformed into 0-handles. In particular the handle structure produced in Lemma 3.18 is based on components of $\partial M^{\prime}$ and essential annuli. Given a non-elementary $M$ with $\partial M$ a union of tori, it is an exercise to reduce any $(M, B, \Delta)$ to $\left(M^{\prime}, B^{\prime}, \Delta^{\prime}\right)$, where $M^{\prime}$ is non-elementary with $\partial M^{\prime}$ a union of tori, $B^{\prime}$ a union of components of $\partial M^{\prime}$ and essential annuli and $C\left(\Delta^{\prime}\right) \leq C(\Delta)$.

Lemma 4.3. Let $M$ be non-elementarily embedded in the compact hyperbolic 3manifold $N$ with $\partial M$ a union of tori and let $(M, B, \Delta)$ be a general based handle structure. Then there exists a general based handle structure $\left(M^{\prime}, B^{\prime}, \Delta^{\prime}\right)$, with $M^{\prime}$ hyperbolic and non-elementary, $\rho_{1}\left(\Delta^{\prime}\right) \leq \rho_{1}(\Delta)$ and $\Delta^{\prime}$ has no 0 -handles.

Proof. Proof by induction on $\rho_{1}(\Delta)$. By Lemma 3.6 we can assume that $M$ is irreducible and hence by Lemma 1.11 that $\partial M$ is incompressible. If $M$ is not hyperbolic, then by [JS], [Jo] there exists a embedded surface $S$ consisting of tori and incompressible, boundary incompressible annuli such that the manifold $M_{1}$ obtained by splitting along $S$ is a union of hyperbolic manifolds and Seifert fibered spaces. By Lemma 1.10 one of these components $M_{2}$ is non-elementary in $N$ and hence hyperbolic.

Let $F$ be a normal surface isotopic to $S$. Let $\left(M_{1}, B_{1}, \Delta_{1}\right)$ be obtained by splitting $(M, B, \Delta)$ along $F$. Let $\left(M_{2}, B_{2}, \Delta_{2}\right)$ be obtained by restricting $\Delta_{1}$ to $M_{2}$. As in $[\mathrm{Mv} 1], \rho_{1}\left(\Delta_{2}\right) \leq \rho_{1}(\Delta)$.

If $B$ contains annuli, and $\partial F$ crosses $B$ in arcs, then $B_{1}$ will contain discs. If $D$ is a disc component of $B_{2}$, then delete it by viewing $D \times I$ as a 0 -handle. Now cancel 0 -handles with 1 -handles to produce $\left(M_{3}, B_{3}, \Delta_{3}\right)$ which either has no 0 -handles or is classical. Note that $\rho_{1}\left(\Delta_{3}\right) \leq \rho_{1}(\Delta)$. If $\Delta_{3}$ is classical, then hollow out a valence $\geq 31$-handle and the 0 -handle to produce $\left(M_{4}, B_{4}, \Delta_{4}\right)$ with $\rho_{1}\left(\Delta_{4}\right)<\rho_{1}\left(\Delta_{3}\right)$. The result follows by induction. 
Lemma 4.4. If $M$ is a compact hyperbolic 3-manifold and if $(M, B, \Delta)$ is a handle structure, then there exists a general based Mom- $k$ structure $\left(M, B^{\prime}, \Delta^{\prime}\right)$ with $\rho_{1}\left(\Delta^{\prime}\right) \leq \rho_{1}(\Delta)$. In particular, if $(M, B, \Delta)$ is a weak Mom-n structure, then $k \leq n$.

Proof. If $\Delta_{1}$ has compressible lakes or valence-0 2-handles, then use Lemma 3.3 to eliminate them and obtain $\left(M, B_{1}, \Delta_{1}\right)$ with $C\left(\Delta_{1}\right)<C(\Delta)$. Note that $M$ is unchanged since $\partial M$ is incompressible. Next, if possible, cancel the valence $\leq 11$ and 2-handles to strictly reduce $C$. If $\sigma$ is a valence-2 2-handle which goes over distinct 1handles, then cancelling a 1 -handle with $\sigma$ creates $\left(M, B, \Delta_{2}\right)$ with $C\left(\Delta_{2}\right)<C\left(\Delta_{1}\right)$.

We now assume that no valence-2 2-handle $\sigma$ goes over distinct 1-handles. Suppose that $\sigma$ goes over the same 1-handle $\alpha$ twice. Then $\sigma \cup \alpha$ can be viewed as an embedded annulus or Möbius band $A$ with boundary on $B$. Since $M$ is hyperbolic and orientable, $A$ must be an annulus and is either boundary parallel in $M$ or compressible in $M$.

If $A$ is boundary parallel, then it together with an annulus on $B$ bound a solid torus $V \subset M$. This contradicts the fact that $\Delta_{2}$ has no 3-handles. Similarly, If $A$ is compressible in $M$, then since $\partial M$ is incompressible and $M$ is irreducible, there exists a 3-ball $V$ in $M$ bounded by $A$ together with two discs in $\partial M$. This again implies that $\Delta_{2}$ has 3-handles.

Thus every 1-handle of $\Delta_{2}$ is valence $\geq 2$ and every 2-handle is valence $\geq 3$. By subdividing the 2-handles we obtain a general based Mom structure $\left(M, B_{3}, \Delta_{3}\right)$ with $\rho_{1}\left(\Delta_{3}\right)=\rho_{1}\left(\Delta_{2}\right) \leq \rho_{1}(\Delta)$. Recall that if $(M, B, \Delta)$ is a general based Mom- $n$ structure, then $n=\rho_{1}(\Delta)$.

Lemma 4.5. If $(M, B, \Delta)$ is a general based internal Mom-n structure on a compact hyperbolic 3-manifold $N$, then $n \geq 2$.

Proof. This follows by direct calculation.

Lemma 4.6. If the compact hyperbolic 3-manifold $N$ has a general based internal Mom-2 structure $(M, B, \Delta)$, then it has a full internal Mom-2 structure.

Proof. By splitting along annuli in $B \times I$ we can assume that every non-peripheral lake of $B$ is a disc.

Suppose $B$ contains two tori $T_{1}$ and $T_{2}$. If each 1-handle connects $T_{1}$ to $T_{2}$, then this implies that each 2-handle is of even valence which is a contradiction. If neither 1-handle connects $T_{1}$ to $T_{2}$ then $M$ is disconnected and consists of either two general based internal Mom-1 structures, contradicting Lemma 4.5, or a Mom-2 structure and a thickened torus, in which case we throw away the torus and reduce to a simpler case. If one 1-handle connects $T_{1}$ to $T_{2}$ and the other connects $T_{1}$ to itself, then the 1-handle connected to $T_{2}$ must have valence at least 4 , since there must be at least two bridges on $T_{2} \times\{1\}$ in order for all of the lakes to be discs. The only possibility 
is that that 1-handle has valence exactly 4 , while the other 1-handle has valence 2 ; in other words, $\Delta$ has a single sheet of valence 4 which is a thickened disc. Now note that $T_{2} \times\{1\}$ has a single island and two bridges, and that those bridges must follow paths which form a meridian-longitude pair for $T_{2} \times\{1\} . T_{1} \times\{1\}$ has three islands and four bridges, but two of those islands only meet the ends of two bridges. We can ignore those islands (i.e., consider the 1-handle of valence 2 to just be part of the sheet) in which case we get the same picture on $T_{1} \times\{1\}$ : one island and two bridges forming a meridian-longitude pair. Now consider the four attaching zones $\alpha_{i} \times I$ where the sheet intersects the boundary of the 1-handle of valence 4 . Together with the four bridges, these regions should join together to form the transverse boundary of the sheet (i.e., the closure of the complement of the lateral boundary) but under the conditions described the union of the attaching zones with the four bridges will have two distinct components, a contradiction.

If $B$ consists of a single torus, then $(M, B, \Delta)$ is a full Mom-2 structure. We finally assume that $B$ contains an annulus. We only discuss the case that $B$ is connected; the case where $B$ is either the union of an annulus and torus or two annuli is similar and easier.

Note that $B \times\{1\}$ contains four islands and six bridges. Let $\alpha$ be a path from one component of $\partial B \times\{1\}$ to the other that crosses no islands and a minimum number of bridges, and let $U$ be the union of the islands, bridges, and non-peripheral lakes. By Lemmas 4.3 and 4.4 we may assume that $M$ is hyperbolic with incompressible boundary, and hence $U$ is a connected essential annulus. Thus $\chi(U)=0$ and hence $U$ has exactly two non-peripheral lakes. Clearly $\alpha$ has to cross each non-peripheral lake at most once, so $\alpha$ crosses at most three bridges. Let $D=\alpha \times I ; D$ is an essential compressing disc for $B \times I$ which cuts across the bridges in at most three components.

Now suppose that $\Delta$ has a single sheet of valence four, i.e., one of the 1-handles of $\Delta$ has valence four and the other has valence two. Let $\eta$ denote the valence-4 1-handle of $\Delta$. View $N(D)$ as a 1-handle and $B \times I-N(D)$ as a 0 -handle to obtain a classical handle structure with two 1 -handles respectively of valence 4 and $\leq 3$. Now as in Lemma 3.11 hollow out the 0 -handle and $\eta$ to get a non-elementary $M_{1}$ with handle structure $\Delta_{1}$ based on a torus with a single 1-handle of valence $\leq 3$. Thus we obtain a Mom-1 structure on a hyperbolic 3-manifold, which is a contradiction.

The other case is that $\Delta$ has two valence-3 1-handles. If $D$ happens to cut the bridges in $\leq 2$ components, then as above we obtain a handle structure with one 0 -handle and three 1-handles. Hollowing out a valence-3 1-handle and the 0-handle produces a handle structure $\Delta_{1}$ on a non-elementary manifold $M_{1}$ with $\rho_{1}\left(\Delta_{1}\right) \leq 1$, which is a contradiction. However it is possible that $D$ cuts the bridges in exactly three components; then we have to look deeper. View $N(D)$ as a 1-handle and $B \times I-N(D)$ as a 0 -handle as before to obtain a handle structure with a 0-handle, 
three 1-handles all of valence 3, and two 2-handles. Denote the original 1-handles by $a$ and $b$ and the new 1-handle by $c$. Since $c$ has valence 3, one of the 2-handles must run over $c$ at least twice; call this 2-handle $\sigma$, and call the other 2-handle $\tau$. Since $\tau$ has valence 3 in $\Delta, \tau$ must run over one of the original 1-handles at least twice; without loss of generality assume $\tau$ runs over $a$ at least twice. Hence $\tau$ runs over $b$ at most once, and it runs over $c$ at most once. If we drill out $a$ and the 0 -handle as before, we will get a non-elementary $M_{1}$ with handle structure $\Delta_{1}$ based on a torus with two 1-handles $b$ and $c$ each of valence 3 and two 2-handles $\sigma$ and $\tau$, where $\tau$ has valence at most 2 . If the valence of $\tau$ is less than 2 we can use the results of the previous section to produce a simpler structure and get a Mom- $k$ structure on $N$ with $k<2$, a contradiction since $N$ is hyperbolic. If the valence of $\tau$ is exactly 2 , then $\tau$ must run over $b$ once and $c$ once, while $\sigma$ runs over $b$ twice and $c$ twice. Cancelling $\tau$ with either $b$ or $c$ results in a new Mom-2 structure on $N$ with a single disc sheet of valence 4. If this new Mom-2 structure is full then we are done, otherwise we can split along annular lakes in the new Mom-2 and reduce the problem to the previous case. Either way, the proof is complete.

Remark 4.7. The figure- 8 knot complement contains a non-full Mom-2 structure with two 1-handles of valence 3 , in addition to having a full Mom-2 structure. Transforming this non-full Mom-2 structure as in the proof of the preceding lemma results in a full Mom-2 structure with a single disk sheet of valence 4 . See $\$ 7$.

Proof of Theorem 4.1. The proof is by induction on $\rho_{1}(\Delta)$. If $M$ is not hyperbolic, then apply Lemma 3.18 to obtain $\left(M_{1}, B_{1}, \Delta_{1}\right)$ which is either a full internal Mom- $k$ structure with $k<n$ (and hence $k=\rho_{1}\left(\Delta_{1}\right)<\rho_{1}(\Delta)=n$ ) or $\rho_{1}\left(\Delta_{1}\right) \leq \rho_{1}(\Delta)-2$ and $M_{1}$ is non-elementary and $\partial M_{1}$ is a union of tori. In the former case the proof follows by induction. In the latter case apply Lemma 4.3 to obtain $\left(M_{2}, B_{2}, \Delta_{2}\right)$ with $M_{2}$ hyperbolic and non-elementary and then apply Lemma 4.4 to obtain $\left(M_{3}, B_{3}, \Delta_{3}\right)$ with $\rho_{1}\left(\Delta_{3}\right) \leq \rho_{1}\left(\Delta_{1}\right)$. If $\rho_{1}\left(\Delta_{3}\right)=2$, then apply Lemma 4.6.

\section{Enumeration of hyperbolic Mom-n's for $2 \leq n \leq 4$}

Let $(M, T, \Delta)$ be a full hyperbolic Mom- $n$, with $2 \leq n \leq 4$. The handle structure $\Delta$ collapses to a cellular complex $K$ in the following fashion. Each 1-handle collapses to the arc at its core, and each 2-handle collapses to the disc at its core (expanded as necessary so that it is still attached to the cores of the appropriate 1-handles). Also, $T \times I$ collapses to $T \times\{1\}$, subdivided into 0 -cells, 1-cells, and 2-cells corresponding to the islands, bridges, and lakes of $(M, T, \Delta)$. (Note that if $(M, T, \Delta)$ were not full, we might have a non-simply connected lake and $K$ would not be a proper cellular complex.) 
The resulting complex $K$ is a spine for $M$ in the sense of [MF]. If all of the 1-handles of $\Delta$ are of valence 3 , then it is also a special spine in the sense of [MF]; however $K$ is not a special spine in general. In particular, in a special spine the link of each point is either a circle or a circle with two or three radii, but if $\Delta$ has a 1-handle of valence $n$ then the endpoints of the corresponding arc in $K$ will have links which are a circle with $n$ radii. This, however, is the only way in which $K$ fails to be a special spine.

In Section 2 of [MF] Matveev and Fomenko describe how a manifold with a special spine can be reconstructed by gluing together truncated or ideal simplices dual to the vertices of the spine. This construction is easily generalized to our situation, and shows that $M$ can be reconstructed from $K$ by gluing together ideal polyhedra dual to the vertices of $K$. The result is an ideal cellulation of $M$ which is dual to the cellular complex $K$.

The 3-cells of this cellulation will be dual to the elements of $K^{0}$, which consist of the endpoints of the cores of the 1-handles of $\Delta$. In addition, since we have assumed each 1-handle of $\Delta$ meets at least two 2-handles, each point $v \in K^{0}$ will be the endpoint of at least two curves in $T \times\{1\} \cap K^{1}$. Hence if $n_{v}$ is the valence of $v$ in the 1 -skeleton of $K$ then $n_{v} \geq 3$. If $n_{v} \geq 4$ then $v$ is dual to an $\left(n_{v}-1\right)$-sided pyramid: the base of the pyramid is dual to the core of a 1-handle while the sides are dual to curves in $T \times\{1\} \cap K^{1}$. If $n_{v}=3$ then $v$ is dual to a "digonal pyramid", which we eliminate from the cellulation by collapsing it to a face in the obvious fashion. Thus $K$ is dual to a cellulation of $M$ by ideal pyramids. Since the bases of these pyramids correspond to the ends of the 1-handles of $\Delta$, we can pair them up into a collection of ideal dipyramids.

We can say more concerning the possible types and combinations of dipyramids. On one hand, each vertex $v$ is adjacent to $n_{v}-1$ edges in $T \times\{1\} \cap K^{1}$, and each such edge has two endpoints; on the other hand, the core of each 2-cell of $\Delta$ contributes three edges to $T \times\{1\} \cap K^{1}$, and there are $n$ such cores in a Mom- $n$. Therefore $\Sigma_{v}\left(n_{v}-1\right)=6 n$ in a Mom- $n$. Furthermore, $n_{v}-1$ must be at least 2 and (if it is greater than 2) equals the number of sides of the pyramid dual to $v$. Finally the vertices $v$ occur in pairs since each one corresponds to an end of a 1-handle, and the vertices in each pair have the same valence. Therefore for a Mom-2, there are only two possibilities: four three-sided pyramids, which glue together to form two three-sided dipyramids, or two four-sided pyramids and two "digonal pyramids", which (after eliminating the "digonal pyramids") glue together to form a single ideal octahedron. Similarly, there are only three possibilities for a Mom-3: three three-sided dipyramids, a three-sided dipyramid together with an octahedron, or a five-sided dipyramid by itself. The five possibilities for a Mom-4 are as follows: four three-sided dipyramids, two three-sided dipyramids and an octahedron, one three-sided dipyramid and one five-sided dipyramid, two octahedra, or one six-sided dipyramid. 
Thus, if $(M, T, \Delta)$ is a hyperbolic Mom-2, Mom-3, or Mom-4 then $M$ can be obtained by gluing together the faces of one of these ten sets of ideal polyhedra. Enumerating the possibilities for $M$ then becomes a matter of enumerating the ways in which the faces of these polyhedra can be glued together to form a hyperbolic 3-manifold.

This task is simplified somewhat by the following observation: the faces of each dipyramid always have exactly one vertex which is dual to the cusp neighborhood $T \times[0,1)$. When gluing the polyhedra together to form $N$, all such vertices must be identified with one another and with no other vertices. Thus given any two faces, there is only one possible orientation-preserving way that those two faces could be glued together.

Hence it is sufficient to enumerate the number of ways in which the faces of one of the ten sets of polyhedra can be identified in pairs. Although it is almost trivial to program a computer to do this, care must be taken as the number of possibilities is a factorial function of the number of faces, and a naive approach can rapidly exhaust a computer's memory. To reduce the demands on the computer, a refinement to the naive approach was employed. First, for each possible set of polyhedra a symmetry group was computed. Each dipyramid has dihedral symmetry, while if a given set of polyhedra contains two dipyramids with the same number of sides then they can be exchanged to provide an additional symmetry. Secondly, an ordering was chosen for the set of all possible pairings of faces, namely the lexicographic ordering of the pairings when represented as permutations. Our computer program considered the set of pairings in order, and any pairing was immediately rejected if it was conjugate to a previous pairing via an element of the symmetry group. This considerably reduced the running time of the program.

The next step in the process is to eliminate pairings which result in obviously non-hyperbolic manifolds. While the program SnapPea can in principle handle this, for reasons of speed our program checked one necessary criterion itself: whether the link of every ideal vertex was Euclidean. Computing the Euler characteristic of the link of each ideal vertex in the cellular complex resulting from a pairing was easy to do and eliminated many cases from consideration. Our program also eliminated any pairing in which the vertices supposedly dual to the original cusp neighborhood or solid torus in fact glued together to form two or more ideal vertices.

The above considerations resulted in a list of gluing descriptions of 4236 manifolds which might be hyperbolic Mom-2's or Mom-3's. At this point, SnapPea was employed to try and compute hyperbolic metrics for each of these manifolds, and to find further hyperbolic symmetries among the manifolds which admitted such metrics. SnapPea claimed to identify 22 hyperbolic manifolds, although only 21 were unique; the manifold known as s785 in the SnapPea census appeared twice. (There were also two cases in which SnapPea experienced an error and was unable to make a determination. In both of these cases, computation by hand showed that the funda- 
mental group of the manifold was isomorphic to the group $\left\langle a, b \mid\left[a, b^{3}\right]\right\rangle$ which has a non-trivial center and hence is not the fundamental group of a hyperbolic manifold with torus boundary components.)

Some comments about rigor are in order here. Since SnapPea relies on floatingpoint arithmetic, some of its results are unavoidably inexact. In particular, there is no guarantee that SnapPea will find a hyperbolic metric on a manifold even if one exists, or that SnapPea will correctly discern the absence of a hyperbolic metric in cases where it does not exist. In practice it is our experience that if one is careful to allow SnapPea to simplify a triangulation before attempting to find a metric, then if a metric exists SnapPea will either find it or fail to make a determination, while if a metric does not exist SnapPea will either correctly say so or on rare occasions "find" a metric with absurdly low volume due to floating-point error. Still, from a standpoint of rigor this is problematic. Fortunately there is at least one task which SnapPea does perform exactly, and that is finding isometries between two different cusped manifolds: SnapPea will only report that an isometry exists if it finds identical triangulations of the two manifolds. (See [We]; in particular see the comments in the source code file isometry.c.) This is a combinatorial operation, not a floating-point one, and hence we are confident that SnapPea performs this operation rigorously.

Those familiar with SnapPea's source code may object that SnapPea re-triangulates each manifold before determining if an isometry exists, and that SnapPea uses floating-point information to choose the re-triangulation. To this objection we would reply that while floating-point information is used to choose the re-triangulation, the actual re-triangulating is still a combinatorial operation, i.e., it uses integer arithmetic. The new triangulation is guaranteed to have the same topological type (see the comments in canonize_part_l.c from [We]), and hence the possibility of floating-point error does not invalidate the result when SnapPea reports that it has found an isometry.

Thus while we are trusting SnapPea when it says that the manifolds mentioned above are all in the isometry class of one of 21 manifolds from the SnapPea census, we are confident that we are not sacrificing rigor in so doing. Furthermore, the census manifolds were recently confirmed to be hyperbolic by Harriet Moser in [Mos], establishing that we have found 21 different hyperbolic Mom-2's and Mom-3's.

Unfortunately, we still cannot trust SnapPea when it fails to find an hyperbolic metric for a given manifold, as that result is not guaranteed to be rigorous. Fortunately SnapPea does have facilities to determine when two triangulations are combinatorially identical; this operation significantly reduced the number of manifolds to consider, and it uses no floating-point arithmetic and hence can be considered rigorous. Doing this results in a list of 66 unique triangulations from the above list of 4236 which need to be confirmed to be non-hyperbolic manifolds. These manifolds were analyzed by hand in the same way as the two manifolds for which SnapPea experienced an error. Namely, SnapPea was used as before to compute the fundamental groups of the manifolds in question, and then we examined the list of groups to see if any of them 
might be the fundamental group of a hyperbolic manifold. The majority of the groups on the list either had a non-trivial center, or else had two rank-2 Abelian subgroups which intersected in a rank-1 Abelian subgroup (also impossible in the fundamental group of a hyperbolic 3-manifold). Some of the groups required further analysis but were still eventually rejected; for example, some groups had an index-two subgroup with one of the above properties even when it was not clear that the whole group had such properties. In the end the hand analysis did not reveal any new hyperbolic 3manifolds in the list of gluing descriptions. This completes the proof of the following result:

Theorem 5.1. There are 3 hyperbolic manifolds $M$ such that $(M, T, \Delta)$ is a Mom-2 for some $T$ and $\Delta$ : the manifolds known in SnapPea's notation as $\mathrm{m} 125, \mathrm{~m} 129$, and $\mathrm{m} 203$. There are 18 additional hyperbolic manifolds $M$ such that $(M, T, \Delta)$ is a Mom-3 structure for some $T$ and $\Delta$ : the manifolds known in SnapPea's notation as m202, m292, m295, m328, m329, m359, m366, m367, m391, m412, s596, s647, s774, s776, s780, s785, s898, and s959.

Remark 5.2. The computer code and data in the preceding description are available from one of the authors [M2].

Some comments about the above list are in order. The manifold $\mathrm{m} 129$, better known as the complement of the Whitehead link, is the only manifold on this list which is obtained by gluing together the faces of an ideal octahedron. Also, all but one of these manifolds have two cusps. The exception is the three-cusped s776, which is the complement in $S^{3}$ of a three-element chain of circles (the link $6_{1}^{3}$ in Rolfsen's notation).

Enumerating hyperbolic Mom-4's was more difficult: merely enumerating the possible gluing descriptions resulted in a list of 1033612 possibilities (compared to 4236 possibilities in the previous case). However, using a similar process to the one described above SnapPea identifies 138 different hyperbolic manifolds. Note that all of the Mom-2's and Mom-3's appear in the Mom-4 list; the same manifold can admit multiple handle structures.

Based on the above result, we propose the following:

Conjecture 5.3. There are 138 hyperbolic manifolds $M$ such that $(M, T, \Delta)$ is a Mom-2, Mom-3, or Mom-4 for some $T$ and $\Delta$. Of these, 117 are strict Mom-4's, i.e., Mom-4's which are not Mom-2's or Mom-3's.

Of the 117 strict Mom-4's, SnapPea was successfully used to identify 83 of them as manifolds from the SnapPea census. Those manifolds appear in Figure 1. SnapPea was not able to identify the remaining 34 manifolds, and in fact 33 of those manifolds have volumes which do not appear anywhere in the SnapPea census, presumably 


\begin{tabular}{|c|c|c|c|c|c|c|}
\hline \hline $\mathrm{m} 357$ & $\mathrm{~s} 579$ & $\mathrm{~s} 883$ & $\mathrm{v} 2124$ & $\mathrm{v} 2943$ & $\mathrm{v} 3292$ & $\mathrm{v} 3450$ \\
$\mathrm{~m} 388$ & $\mathrm{~s} 602$ & $\mathrm{~s} 887$ & $\mathrm{v} 2208$ & $\mathrm{v} 2945$ & $\mathrm{v} 3294$ & $\mathrm{v} 3456$ \\
$\mathrm{~s} 441$ & $\mathrm{~s} 621$ & $\mathrm{~s} 895$ & $\mathrm{v} 2531$ & $\mathrm{v} 3039$ & $\mathrm{v} 3376$ & $\mathrm{v} 3468$ \\
$\mathrm{~s} 443$ & $\mathrm{~s} 622$ & $\mathrm{~s} 906$ & $\mathrm{v} 2533$ & $\mathrm{v} 3108$ & $\mathrm{v} 3379$ & $\mathrm{v} 3497$ \\
$\mathrm{~s} 503$ & $\mathrm{~s} 638$ & $\mathrm{~s} 910$ & $\mathrm{v} 2644$ & $\mathrm{v} 3127$ & $\mathrm{v} 3380$ & $\mathrm{v} 3501$ \\
$\mathrm{~s} 506$ & $\mathrm{~s} 661$ & $\mathrm{~s} 913$ & $\mathrm{v} 2648$ & $\mathrm{v} 3140$ & $\mathrm{v} 3383$ & $\mathrm{v} 3506$ \\
$\mathrm{~s} 549$ & $\mathrm{~s} 782$ & $\mathrm{~s} 914$ & $\mathrm{v} 2652$ & $\mathrm{v} 3211$ & $\mathrm{v} 3384$ & $\mathrm{v} 3507$ \\
$\mathrm{~s} 568$ & $\mathrm{~s} 831$ & $\mathrm{~s} 930$ & $\mathrm{v} 2731$ & $\mathrm{v} 3222$ & $\mathrm{v} 3385$ & $\mathrm{v} 3518$ \\
$\mathrm{~s} 569$ & $\mathrm{~s} 843$ & $\mathrm{~s} 937$ & $\mathrm{v} 2732$ & $\mathrm{v} 3223$ & $\mathrm{v} 3393$ & $\mathrm{v} 3527$ \\
$\mathrm{~s} 576$ & $\mathrm{~s} 859$ & $\mathrm{~s} 940$ & $\mathrm{v} 2788$ & $\mathrm{v} 3224$ & $\mathrm{v} 3396$ & $\mathrm{v} 3544$ \\
$\mathrm{~s} 577$ & $\mathrm{~s} 864$ & $\mathrm{~s} 941$ & $\mathrm{v} 2892$ & $\mathrm{v} 3225$ & $\mathrm{v} 3426$ & $\mathrm{v} 3546$ \\
$\mathrm{~s} 578$ & $\mathrm{~s} 880$ & $\mathrm{~s} 948$ & $\mathrm{v} 2942$ & $\mathrm{v} 3227$ & $\mathrm{v} 3429$ & \\
\hline \hline
\end{tabular}

Figure 1. Conjectured list of SnapPea manifolds which are strict Mom-4's.

because the Matveev complexity of the corresponding manifolds is greater than 7 (see $[\mathrm{MF}]$ ). The remaining manifold has the same volume and homology as the census manifold v3527; it is conceivable that SnapPea was simply unable to find a corresponding isometry.

The unidentified manifolds are listed in Figure 2. The notation used can be interpreted as follows: the numbers before the semi-colon describe the type of ideal polyhedra used to construct the manifold. For example, the first entry in the figure has the numbers " $3,3,4$ " to the left of the semi-colon; each " 3 " indicates an ideal triangular dipyramid, while each " 4 " indicates an ideal square dipyramid (i.e., an ideal octahedron). Each ideal dipyramid has two "polar" vertices and either three or four "equatorial vertices". Number the faces of all the polyhedra sequentially in such a way that the faces "north" of each equator are numbered before the faces "south" of each equator. For example, in the first entry the first triangular dipyramid has faces 0,1 , and 2 next to one polar vertex, and faces 10,11 , and 12 next to the other polar vertex. The next triangular dipyramid has faces 3, 4, and 5 as well as faces $13,14,15$, and the square dipyramid has faces 6 through 9 and 16 through 19. (This somewhat unintuitive numbering scheme was chosen for convenience when writing the computer software for this part of the paper.) Then the numbers to the right of the semi-colon form a permutation which describes how to glue together the faces of the ideal polyhedra. For example, in the first entry the string of numbers which begins with " $3,6,8,0, \ldots$ " imply that face 0 is glued to face 3 , face 1 is glued to face 6 , and so on. Since we are requiring "polar" vertices to be identified solely with other "polar" vertices, no other information is needed to reconstruct the polyhedral gluing.

One additional point of information: all but eight of the manifolds in the list satisfy $|\partial M|=2$; seven satisfy $|\partial M|=3$ and one satisfies $|\partial M|=4$. Thanks to 


\begin{tabular}{|c|}
\hline 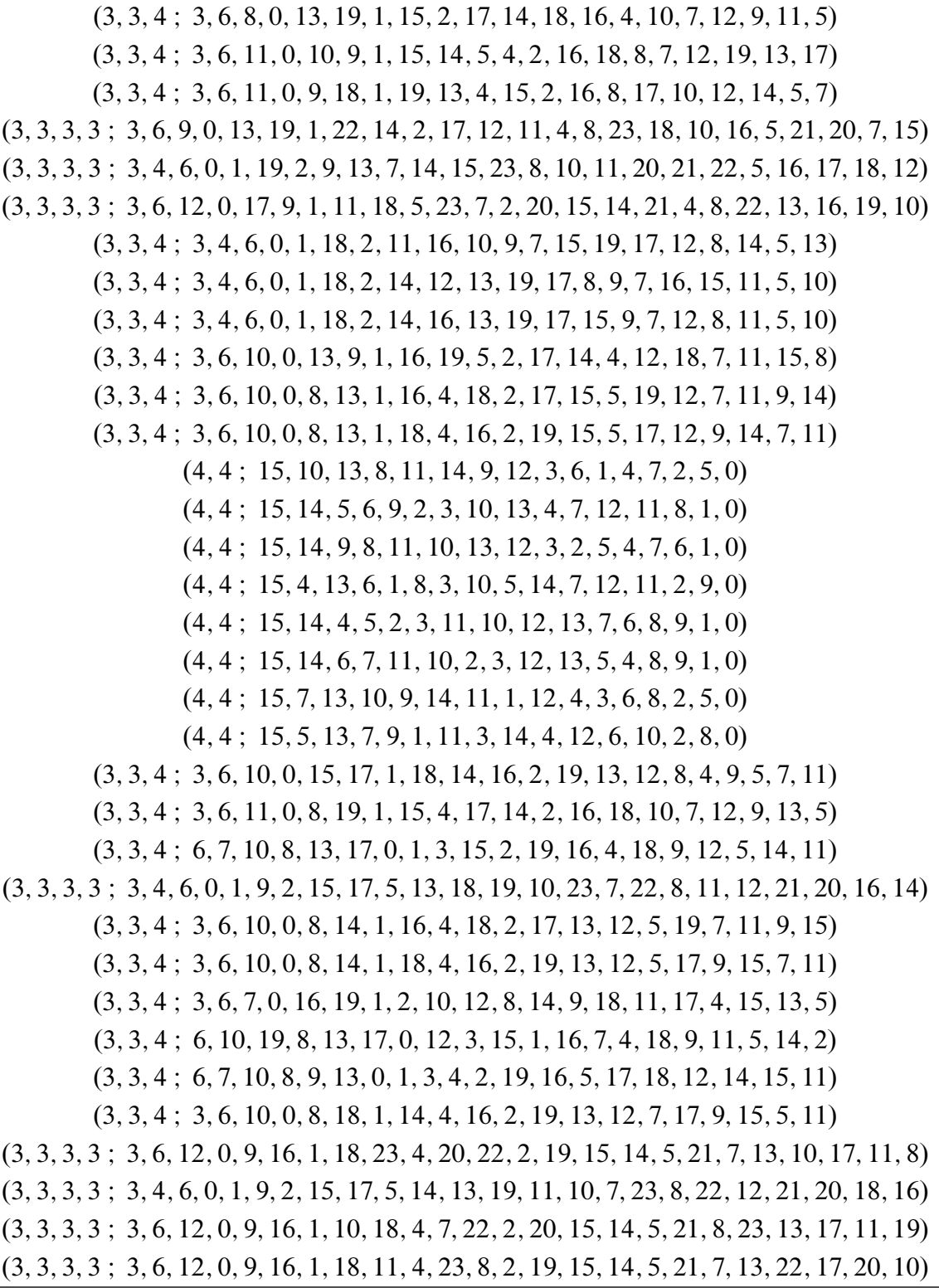 \\
\hline
\end{tabular}

Figure 2. Conjectured Mom-4's not identified by SnapPea.

the timely assistance of Morwen Thistlethwaite, the authors were able to positively identify all eight of these manifolds: 
Conjecture 5.4. There are 8 hyperbolic manifolds $M$ such that $(M, T, \Delta)$ is a Mom$n$ for some $2 \leq n \leq 4$ and $|\partial M|>2$. All eight manifolds are complements of links in $S^{3}$ : the links $6_{1}^{3}, 6_{2}^{3}$ (the Borromean rings), $8_{1}^{3}, 8_{9}^{3}, 8_{2}^{4}$, and the links with Gauss codes jeccddEGHiJBFCa, jcbecceaHbIJDGF, and mccdfiEhAjKLcmdbFG.

At the time of writing we are still searching for an efficient way to verify SnapPea's computations in the Mom-4 case; examining all of the fundamental groups by hand is no longer a practical solution in this case. Until a better way is found, our enumeration results in the Mom-4 case should properly be considered speculative.

\section{For $n \leq 4$, hyperbolic Mom-n's in hyperbolic 3-manifolds are internal Mom-n's}

Let $R$ be a convolotube in the interior of a compact hyperbolic 3-manifold $N$ and let $V$ be the cube with knotted hole bounded by $R$. By drilling out solid tori from $N-\stackrel{\circ}{V}$, we can create a manifold $M$ which is non-elementarily embedded in $N$ and whose boundary contains a convolutube. We call such an embedding knotted. The goal of this chapter is to show that if $n \leq 4$, any embedding of a Mom- $n$ manifold $(M, T)$ into a compact hyperbolic manifold $(N, T)$ is unknotted.

Definition 6.1. Let $M$ be a compact 3-manifold and $T$ a possibly empty union of components of $\partial M$. We say that $(M, T)$ is hereditarily unknotted, if every non-elementary embedding into a compact hyperbolic 3-manifold $N$, taking $T$ to components of $\partial N$, has the property that each component of $\partial M$ is either boundary parallel or bounds a solid torus.

Remark 6.2. If $(M, T)$ is hereditarily unknotted and $M_{1}$ is obtained by filling a component of $\partial M-T$, then $\left(M_{1}, T\right)$ is hereditarily unknotted.

Lemma 6.3. If $(M, T)$ is a hereditarily unknotted Mom- $n$ manifold non-elementarily embedded in the hyperbolic 3-manifold $N$ such that $T$ bounds a tubular neighborhood of a geodesic, then $(M, T)$ is an internal Mom-n structure.

Proof. Let $V$ be the solid torus bounded by $T$. By Lemma 1.9, if $N_{1}=N-\stackrel{\circ}{V}$ with cusp neighborhoods deleted, then $N_{1}$ is compact hyperbolic. Therefore $(M, T) \subset$ $\left(N_{1}, T\right)$ is a non-elementary embedding and hence any component of $\partial M-T$ either bounds a solid torus or is boundary parallel in $N_{1}$. Therefore similar properties hold in $N$ and hence $(M, T)$ is an internal Mom- $n$ structure on $N$.

Remark 6.4. The condition that $T$ bounds a neighborhood of a geodesic is essential. 
Lemma 6.5. Let $M$ be a compact hyperbolic 3-manifold with $T$ a union of components of $\partial M$. If $\partial M-T$ is connected, $(M, T)$ is hereditarily unknotted.

Proof. If under a non-elementary embedding $(M, T) \rightarrow(N, T), \partial M-T$ was a convolutube, then $M$ would be reducible.

The following result establishes criteria for showing that $(M, T)$ is hereditarily unknotted.

Lemma 6.6. Let $M$ be a compact hyperbolic 3-manifold with $V_{1}, \ldots, V_{n}$ components of $\partial M$ and $T$ a nonempty union of some other components. If any of the following hold, there exists no non-elementary embedding $(M, T) \rightarrow(N, T)$ such that $N$ is compact hyperbolic and $\left\{V_{1}, \ldots, V_{n}\right\}$ is exactly the set of convolutubes of $\partial M \subset N$.

i) The manifold obtained by some filling of $M$ along $V_{1}, \ldots, V_{n}$ is a 3-manifold without any hyperbolic part. (That is, after applying sphere and torus decompositions there are no hyperbolic components.)

ii) After some filling of $M$ along $V_{1}, \ldots, V_{n}$, the surface $T$ is compressible.

iii) For every filling on a non-empty set of components of $\partial M-T \cup V_{1} \cup \cdots \cup V_{n}$, either $V_{1} \cup \cdots \cup V_{n}$ is incompressible or the filled manifold has no hyperbolic part.

Proof. Suppose that $(M, T)$ embeds in $(N, T)$, where among the components of $\partial M$, $V_{1}, \cdots, V_{n}$ are the set of convolutubes and $W_{1}, \cdots, W_{m}$ are the tubes. Let $W_{i}^{*}$ denote the solid torus bounded by $W_{i}$ and $V_{i}^{*}$ denote the cube with knotted hole bounded by $V_{i}$. Let $B_{1}, \cdots, B_{n}$ be pairwise disjoint 3-balls in $N$ such that for each $i, V_{i} \subset B_{i}$.

i) Let $\hat{M}$ be a manifold obtained by filling the $V_{i}$ 's. Let $\widehat{N}$ be obtained by deleting the $V_{i}^{*}$ 's and doing the corresponding fillings along the $V_{i}$ 's. Therefore $\hat{N}$ is obtained from $\hat{M}$ by Dehn filling and $\hat{N}$ is a connected sum of $N$ with $S^{2} \times S^{1}$,s and/or lens spaces and/or $S^{3}$ 's. This implies that $\widehat{M}$ has a hyperbolic part.

ii) If $T$ is compressible in $\widehat{M}$ it is compressible in $\widehat{N}$ and hence in $N$, which is a contradiction.

iii) First observe that $V_{i}$ compresses in the manifold $M^{\prime}$ obtained by filling $M$ where each $W_{i}$ is filled with $W_{i}^{*}$. Topologically, $M^{\prime}$ is homeomorphic to $N$ with $n$ open, unknotted, and unlinked solid tori removed and so has a hyperbolic part.

Theorem 6.7. If the Mom- $n$ manifolds for $n \leq 4$ with three or more boundary components are exactly those listed in Figure 3 (i.e., if Conjecture 5.1 is true), then any hyperbolic Mom- $n$ manifold $(M, T)$ with $n \leq 4$ is hereditarily unknotted.

Proof. By Lemma 6.5 it suffices to consider the case where $M$ is one of the eight Mom-4 manifolds with at least three boundary components listed in Figure 3. If $M$ is any of the first six manifolds and $T$ is any component of $\partial M$, then $(M, T)$ 
$6_{1}^{3}$

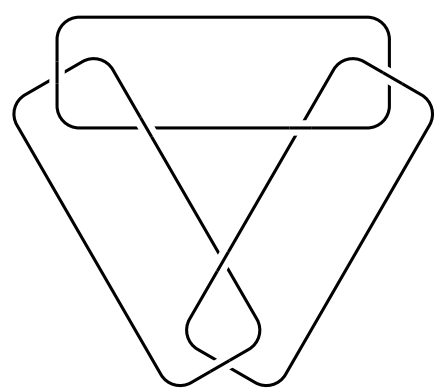

$8_{1}^{3}$

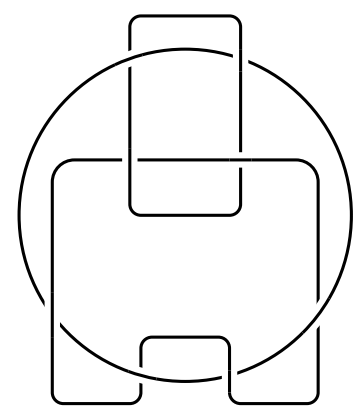

$8_{2}^{4}$

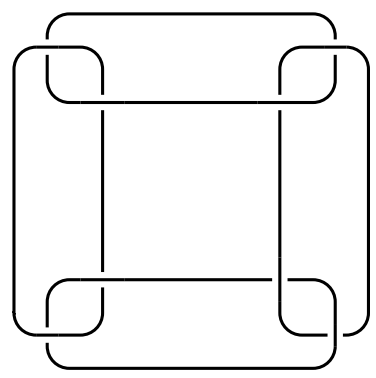

jcbecceaHbIJDGF

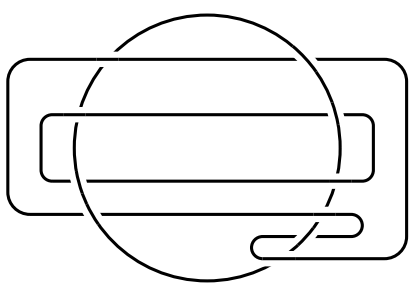

$6_{2}^{3}$

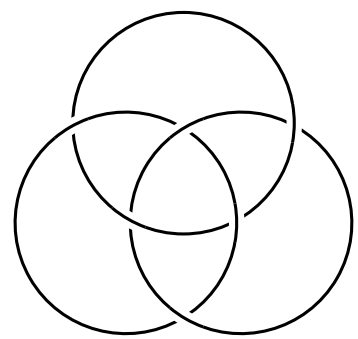

$8_{9}^{3}$

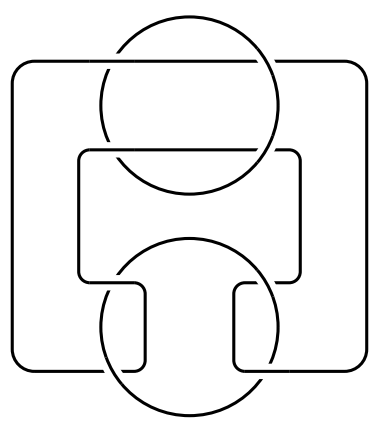

jcccddEGHiJBFCa

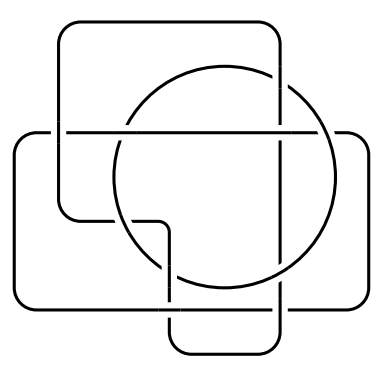

mccdfiEhAjKLcmdbFG

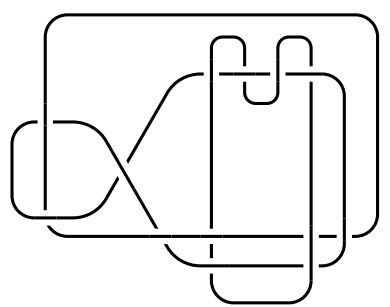

Figure 3. Eight links whose complements are Mom-4's with 3 or more cusps. 
is hereditarily unknotted by criterion (i) of Lemma 6.6. For manifolds 7 and 8 , depending on which boundary component is used for $T$, applications of (i) and (iii) imply that they are hereditarily unknotted.

\section{Examples of Mom- $n$ structures}

In this section we give some representative examples of hyperbolic manifolds $N$ which contain an internal Mom-2 or Mom-3 structure $(M, T, \Delta)$. Our goal in this section is to give the reader an intuitive feel for how these particular cell complexes arise inside hyperbolic manifolds. All of the manifolds in this section involve manifolds $N$ with torus boundary, with the base torus of the Mom-structure $(M, T, \Delta)$ being $\partial N$. To obtain Mom- $n$ structures on closed manifolds, note that if $T=\partial N$ then a Mom- $n$ structure $(M, T, \Delta)$ on $N$ passes to a Mom- $n$ structure on any manifold obtained by filling $\partial N$.

Example 7.1. The first example is the figure-8 knot complement. We construct a Mom-2 $(M, T, \Delta)$ inside this manifold as follows. The torus $T$ is just the boundary of the manifold. The 1-handles span the two tangles which make up the standard diagram of this knot, as seen in Figure 4 (a). Finally the 2-handles are symmetrically placed as shown in Figure 4 (a). Note that, as required, each 2-handle meets three 1-handles counting multiplicity. Specifically, each 2-handle meets the top 1-handle twice and the bottom 1-handle once. Also, one can see from the diagram that the complement of $T \cup\{1$-handles $\} \cup\{2$-handles $\}$ consists of a solid torus, and that the solid torus retracts onto a homotopically non-trivial simple closed curve (which is a geodesic in $N$ ). Thus this is a valid hyperbolic internal Mom-2 structure on $N$.
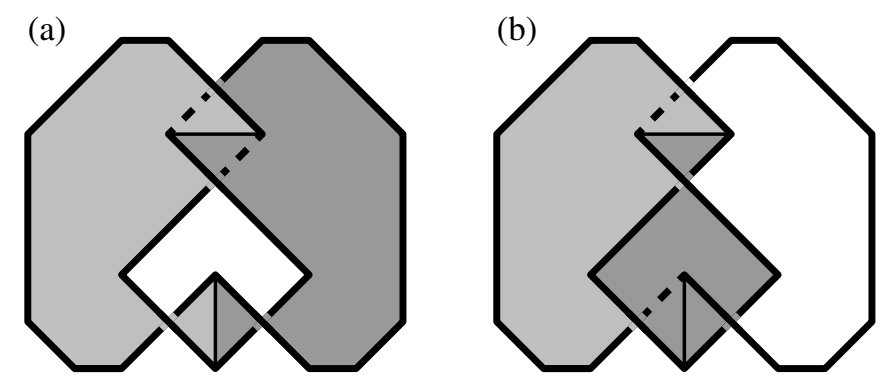

Figure 4. The figure- 8 knot complement equipped with (a) a full internal hyperbolic Mom-2 structure, and (b) a non-full internal Mom-2 structure with a non-hyperbolic underlying submanifold $M$. 
Moreover, we can quickly determine the nature of the ideal triangulation of $M$ described in the Section 5. The ends of the valence-4 1-handle are each dual to a four-sided pyramid in this triangulation, and the two endpoints of the valence-2 1-handle are each dual to a "digonal pyramid", each of which gets flattened to a face and ignored. Thus the figure- 8 knot complement possesses an internal Mom2 structure $(M, T, \Delta)$ where $M$ is a two-cusped hyperbolic manifold which is in turn obtained by gluing together the faces of an ideal octahedron. By the comments after Theorem 5.1, $M$ must be the complement of the Whitehead link. And indeed, it is easy to verify that if one drills out the core of the solid torus in the complement of $M$ one obtains a manifold homeomorphic to the complement of the Whitehead link.

Figure 4 (b) shows a similar internal Mom-2 structure $(M, T, \Delta)$ on $N$, but in this case the structure is not full as the boundary of the rightmost arc in the diagram is an annular lake. Consequently, $M$ in Figure 4 (b) has an embedded essential annulus and is a non-hyperbolic manifold. However we can split $M$ along this essential annulus to obtain $M_{1} \subset N$, and split $T$ to obtain an annulus $B$; then using the same 1-handles and 2-handles we get a full general based Mom-2 structure $\left(M_{1}, B, \Delta_{1}\right)$ on $N$, where $M_{1}$ has a single torus boundary component which is boundary parallel in $N$ (in fact $M_{1}$ is a deformation retract of $N$ ) and where $B$ is a $\pi_{1}$-injective annulus in this boundary component. Transforming this general based Mom-2 structure as in Lemma 4.6 results in another full internal Mom-2 structure on $N$.

Example 7.2. Next we will let $N$ be the manifold known as m003 in the SnapPea census. This manifold has first homology group $\mathbb{Z}+\mathbb{Z} / 5$, and hence is not a knot complement; instead, we will present this manifold as the union of two regular ideal hyperbolic tetrahedra; see Figure 5. Note that in the diagram each face is glued to the corresponding face on the other tetrahedron, in such a way that the edges match up into two equivalence classes as shown. To make $N$ a compact manifold with torus boundary, assume the ideal tetrahedra are truncated. Now suppose that we construct $(M, T, \Delta)$ in this case as follows. For the 1-handles, we use neighborhoods of the two edges shown in the diagram, truncated by the torus $T=\partial N$. And for the 2handles, we use neighborhoods of the two truncated triangles which are formed by gluing together the faces on the front of each tetrahedron in the diagram. It is a simple exercise to confirm that the complement of the resulting embedded manifold $M$ consists of a solid torus, and that the solid torus retracts onto a simple closed geodesic curve, and that therefore this manifold possesses a valid hyperbolic Mom-2 structure. Each of the 1-handles in this Mom-2 meets three of the 2-handles, counting multiplicity; therefore we can conclude that m003 contains a Mom-2 $(M, T, \Delta)$ where $M$ is obtained by gluing together two ideal three-sided dipyramids. From Theorem 5.1 and the comments following it we know this must be either m125 or m203. Further investigation with SnapPea shows that it must in fact be $\mathrm{m} 125$. 

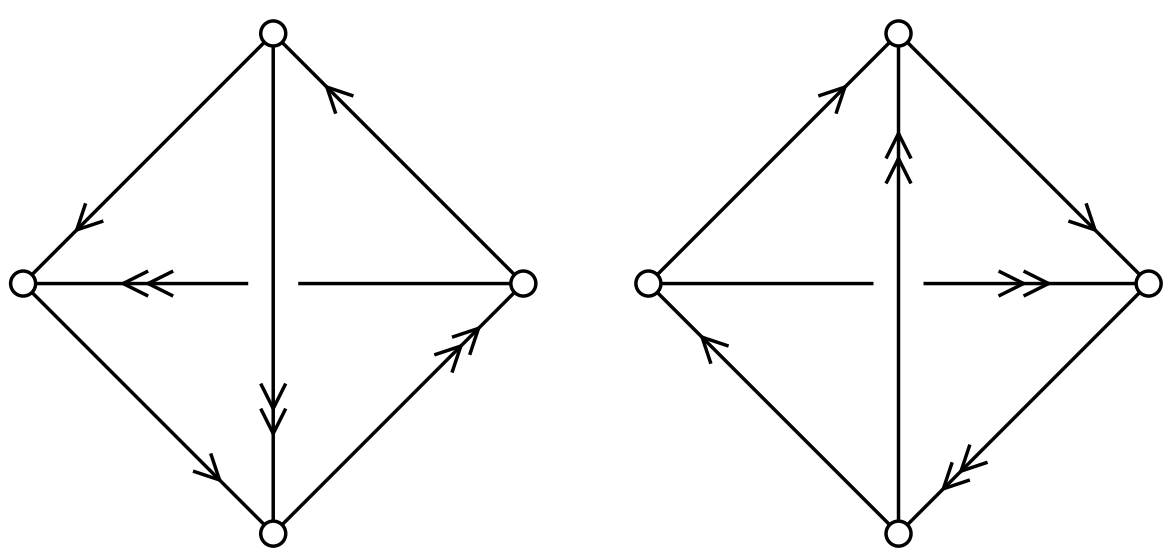

Figure 5. The two ideal tetrahedra making up the manifold m003.

It is instructive to get another view of this Mom-2 by constructing a cusp diagram for this manifold. Specifically, consider the triangulation induced on $T$ by the given ideal triangulation of m003. The two ideal tetrahedra in m003 will appear as eight triangles, the four ideal triangles will appear as twelve edges, and the two edges will appear as four vertices. The resulting cusp diagram is shown in Figure 6; keep in mind this is a diagram of a torus, so the edges of the parallelogram are identified with one another. (The labels inside each triangle indicate which of the ideal simplices

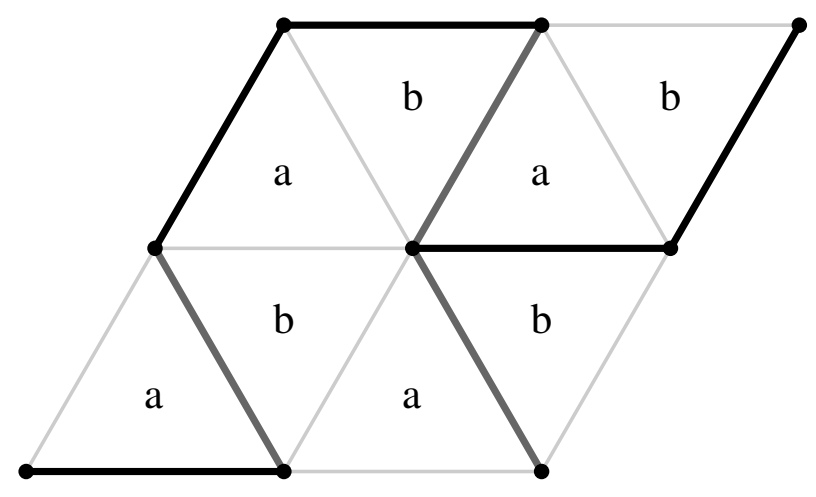

Figure 6. The cusp diagram for m003, with the components of the Mom-2 highlighted.

contributes that triangle to the cusp diagram.) The highlighted edges in the cusp diagram are those that correspond to the 2-handles of the handle structure $\Delta$; in other words, they along with the four vertices of the diagram comprise $\Delta^{1} \cap T$. 
Example 7.3. As another example in this vein, consider the manifold $N=\mathrm{m} 017$. This manifold has first homology group $\mathbb{Z}+\mathbb{Z} / 7$, so again it is not a knot complement in $S^{3}$, but for brevity's sake we only present a cusp diagram here. In Figure 7 , the corners of the three ideal hyperbolic tetrahedra which make up m017 can be seen. And again, the highlighted edges in the cusp diagram correspond to two faces of those tetrahedra which provide the 2-handles for an internal Mom-2 in this manifold. Note that we can determine from the cusp diagram alone that the 1-handles of this Mom-2 meet four and two 2-handles respectively, counting multiplicity, and that therefore in the resulting Mom-2 structure $(M, T, \Delta)$ the manifold $M$ is obtained by gluing together an ideal octahedron. As before, this implies that $M$ must be homeomorphic to the complement of the Whitehead link. Some further work with SnapPea confirms this: m017 is obtained by $(-7,2)$ Dehn surgery on either component of the link.

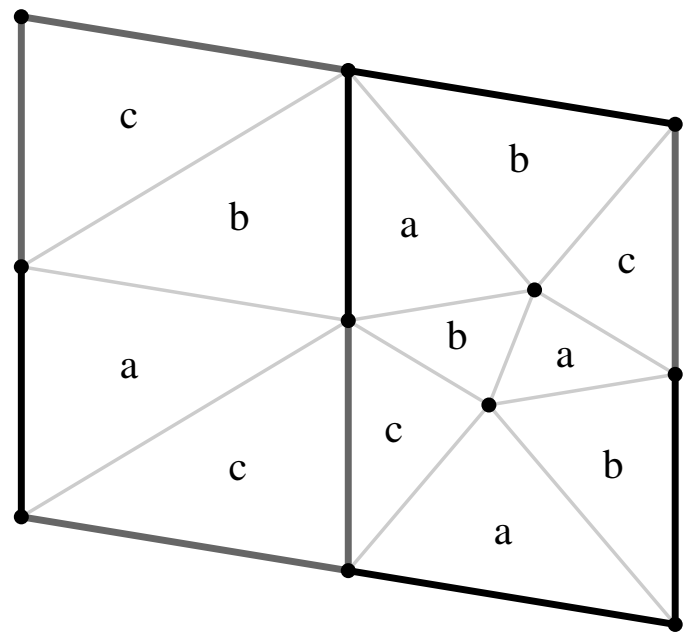

Figure 7. The cusp diagram for m017, with the components of a Mom-2 highlighted.

Example 7.4. Finally, we include the motivating example for this paper. Figure 8 shows the maximal cusp diagram of the 1-cusped manifold m011 as provided by Weeks' SnapPea program. Unlike the previous cusp diagrams in this section, it also shows all the horoballs at hyperbolic distance at most 0.51 from the maximal horoball at infinity. The parallelogram shows a fundamental domain for the $\mathbb{Z} \oplus \mathbb{Z}$ action. Note that the ideal triangulation presented in this diagram is dual to the Ford decomposition of the manifold. In particular the 1-simplices of the triangulation are geodesics orthogonal to pairs of horoballs; these 1-simplices appear either as edges in the figure joining the endpoints of the simplex in $S_{\infty}^{2}$, or as the vertical geodesics passing from the center of each horoball to the horoball at infinity. 


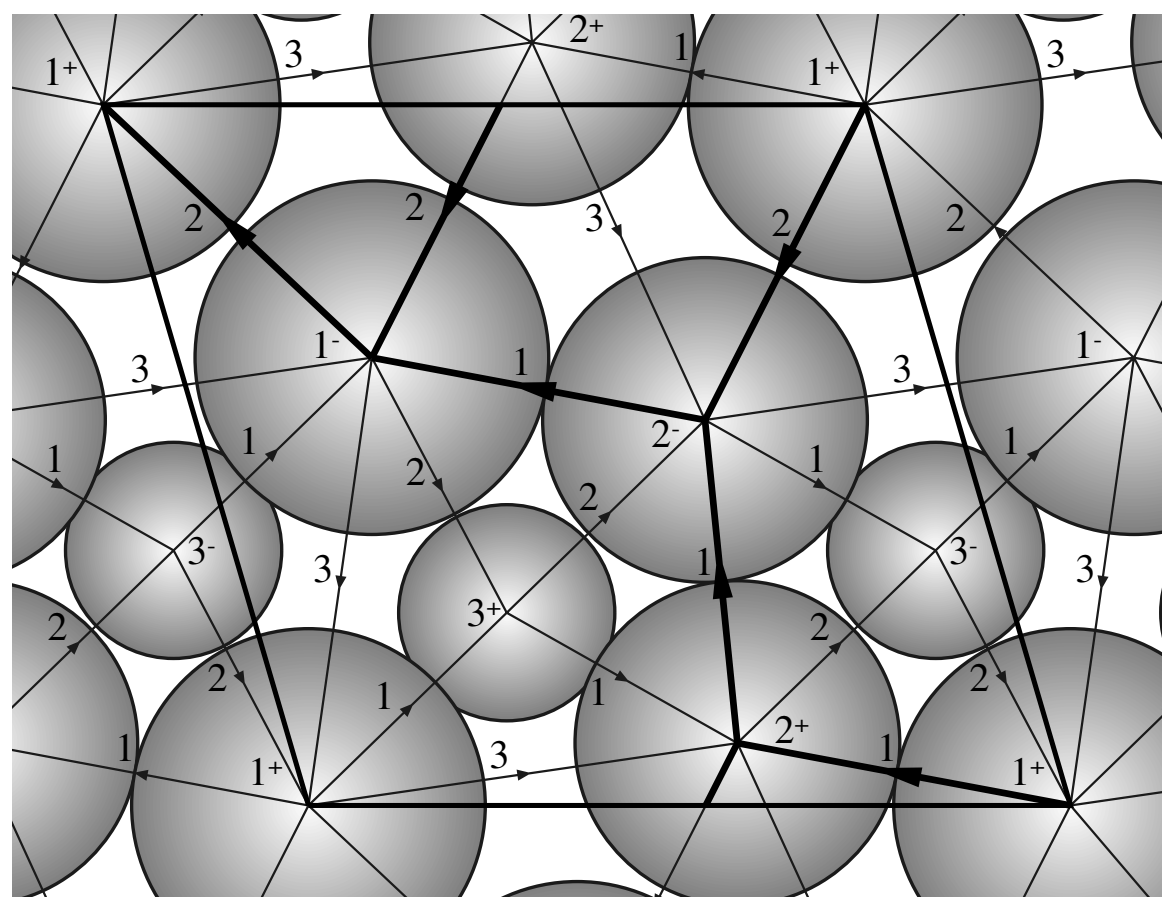

Figure 8. SnapPea's cusp diagram for m011, with the components of a Mom-2 highlighted.

Let $H_{\infty}$ denote the horoball at infinity. There are six horoballs in the diagram up to the $\mathbb{Z} \oplus \mathbb{Z}$-action, labelled 1-, 1+, 2-, 2+, 3-, and 3+. This notation means that if $\gamma \in \pi_{1}(\mathrm{~m} 011)$ takes horoball $n \pm$ to $H_{\infty}$, the horoball at infinity, then $H_{\infty}$ is transformed to one labelled $n \mp$. The geodesic from $n-$ to $H_{\infty}$ is oriented to point into $H_{\infty}$ and hence the geodesic from $n+$ to $H_{\infty}$ is oriented out of $H_{\infty}$. These orientations induce, via the $\pi_{1}(\mathrm{~m} 011)$-action, the indicated orientations on the edges of the diagram. We explain, by example, the meaning of the edge labels. The edge 3 from $2+$ to $2-$ corresponds to a geodesic $\sigma$ with the property that when $2+$ is transformed to $H_{\infty}$, then $2-$ is transformed to $3+$ and $\sigma$ is transformed to the vertical geodesic oriented from $H_{\infty}$ to $3+$. (Had the edge been oriented oppositely, then 2 - would have been transformed to $3-$.) SnapPea did not provide the orientation information, however such information can be derived from the SnapPea data.

By staring at this diagram we can see how m011 contains a Mom-2. Let $V_{0}$ be the maximal horotorus neighborhood of the cusp, slightly shrunken. By expanding $V_{0}$, the expanded $V_{0}$ touches the (expanded) horoballs labeled 1, thereby creating a 1-handle denoted $E_{1}$. Let $V_{1}$ denote this expanded $V_{0}$. Further expansion creates $V_{2}$ which is topologically $V_{1}$ together with another 1-handle $E_{2}$, this 1-handle occurring 
between horoball 2 and $H_{\infty}$. The edge labelled 1 between horoballs $2-$ and $2+$ corresponds to a valence three 2-handle which goes over $E_{1}$ once and $E_{2}$ twice. Similarly the edge labelled 2 between horoballs $1-$ and $1+$ gives rise to a valence three 2-handle going twice over $E_{1}$ and once over $E_{2}$. The parallelogram of Figure 8 can also be viewed as $\partial V_{0}$, with the centers of $1-, 1+, 2-, 2+$ as the attaching sites of the 1-handles and the thick black lines corresponding to where the 2-handles cross over $\partial V_{0}$.

\section{References}

[GM] F. W. Gehring and G. J. Martin, Precisely invariant collars and the volume of hyperbolic 3-folds. J. Differential Geom. 49 (1998), 411-435. Zbl 0989.57010 MR 1669657

[GMM] D. Gabai, R. Meyerhoff, and P. Milley, Volumes of tubes in hyperbolic 3-manifolds. J. Differential Geom. 57 (2001), 23-46. Zbl 1029.57014 MR 71490

[GMM3] D. Gabai, R. Meyerhoff, and P. Milley, Minimum volume cusped hyperbolic 3manifolds. J. Amer. Math. Soc. 22 (2009), no. 4, 1157-1215. Zbl MR 2525782

[Ha] W. Haken, Theorie der Normalflächen. Acta Math. 105 (1961), 245-375. Zbl 0100.19402 MR 0141106

[Jac] W. Jaco, Lectures on three-manifold topology. CBMS Regional Conf. Ser. in Math. 43, Amer. Math. Soc., Providence, R.I., 1980. Zbl 0433.57001 MR 0565450

[JS] W. Jaco and P. Shalen, Seifert fibered spaces in 3-manifolds. Mem. Amer. Math. Soc. 21 (1979), no. 220. Zbl 0415.57005 MR 0539411

[Jo] K. Johannson, Homotopy equivalences of 3-manifolds with boundaries. Lecture Notes in Math. 761, Springer-Verlag, Berlin 1979. Zbl 0412.57007 MR 0551744

[Koj] S. Kojima, Deformations of hyperbolic 3-cone-manifolds. J. Differential Geom. 49 (1998), 469-516. Zbl 0990.57004 MR 1669649

[LM] M. Lackenby and R. Meyerhoff, The maximal number of exceptional Dehn surgeries. Preprint, 2008. arXiv:0808.1176v1 [mathGT]

[MF] S. Matveev and A. Fomenko, Constant energy surfaces of Hamiltonian systems, enumeration of three-dimensional manifolds in increasing order of complexity, and computation of volumes of closed hyperbolic manifolds. Russian Math. Surveys 43 (1988), no. 1, 3-24. Zbl 0671.58008 MR 0937017

[M1] P. Milley, Minimum-volume hyperbolic 3-manifolds. J. Topol. 2 (2009), no. 1, 181-192. Zbl 1165.57016 MR 2499442

[M2] P. Milley, Source code, data, and intermediate results are available at http://www.ems-ph.org/doi/10.4171/CMH/221

[Mos] H. Moser, Proving a manifold to be hyperbolic once it has been approximated to be so. Ph.D. thesis, Department of Mathematics, Columbia University, 2005. Available at http://www.math.columbia.edu/ moser 
[Mv1] S. Matveev, An algorithm for the recognition of 3-spheres (according to Thompson). Mat. Sb. 186 (1995), no. 5, 69-84; English transl. Sb. Math. 186 (1995), no. 5, 695-710.Zbl 0849.57010 MR 1341085

[Mv2] S. Matveev, Algorithmic topology and classification of 3-manifolds. Algorithms Comput. Math. 9, Springer-Verlag, Berlin 2003. Zbl 1048.57001 MR 1997069

[Sch] H. Schubert, Bestimmung der Primfaktorzerlegung von Verkettungen. Math. Z. 76 (1961), 116-148. Zbl 0097.16302 MR 141107

[Th1] W. P. Thurston, The geometry and topology of 3-manifolds. Original 1978 Princeton Notes.

[Th2] W. P. Thurston, Three-dimensional manifolds, Kleinian groups and hyperbolic geometry. Bull. Amer. Math. Soc. 6 (1982), 357-381. Zbl 0496.57005 MR 0648524

[We] J. R. Weeks, SnapPea: A computer program for creating and studying hyperbolic 3-manifolds. Available from http://www.geometrygames.org/

Received October 10, 2008

David Gabai, Department of Mathematics, Princeton University, Princeton, NJ 08544, U.S.A.

E-mail: gabai@princeton.edu

Robert Meyerhoff, Department of Mathematics, Boston College, Chestnut Hill, MA 02467, U.S.A.

E-mail: robert.meyerhoff@bc.edu

Peter Milley, 1107-547 Belmont Avenue West, Kitchener, ON, N2M 5G9, Canada

E-mail: peter.milley@gmail.com 